\title{
The Oct1 homolog Nubbin is a repressor of NF-KB-dependent immune gene expression that increases the tolerance to gut microbiota
}

Widad Dantoft ${ }^{1}$, Monica M Davis ${ }^{1}$, Jessica M Lindvall ${ }^{2}$, Xiongzhuo Tang ${ }^{1}$, Hanna Uvell ${ }^{1,3}$, Anna Junell ${ }^{1}$, Anne Beskow ${ }^{1,4}$ and Ylva Engström ${ }^{1 *}$

\begin{abstract}
Background: Innate immune responses are evolutionarily conserved processes that provide crucial protection against invading organisms. Gene activation by potent NF-KB transcription factors is essential both in mammals and Drosophila during infection and stress challenges. If not strictly controlled, this potent defense system can activate autoimmune and inflammatory stress reactions, with deleterious consequences for the organism. Negative regulation to prevent gene activation in healthy organisms, in the presence of the commensal gut flora, is however not well understood.

Results: We show that the Drosophila homolog of mammalian Oct1/POU2F1 transcription factor, called Nubbin (Nub), is a repressor of NF-kB/Relish-driven antimicrobial peptide gene expression in flies. In nub ${ }^{1}$ mutants, which lack Nub-PD protein, excessive expression of antimicrobial peptide genes occurs in the absence of infection, leading to a significant reduction of the numbers of cultivatable gut commensal bacteria. This aberrant immune gene expression was effectively blocked by expression of Nub from a transgene. We have identified an upstream regulatory region, containing a cluster of octamer sites, which is required for repression of antimicrobial peptide gene expression in healthy flies. Chromatin immunoprecipitation experiments demonstrated that Nub binds to octamer-containing promoter fragments of several immune genes. Gene expression profiling revealed that Drosophila Nub negatively regulates many genes that are involved in immune and stress responses, while it is a positive regulator of genes involved in differentiation and metabolism.
\end{abstract}

Conclusions: This study demonstrates that a large number of genes that are activated by NF-KB/Relish in response to infection are normally repressed by the evolutionarily conserved Oct/POU transcription factor Nub. This prevents uncontrolled gene activation and supports the existence of a normal gut flora. We suggest that Nub protein plays an ancient role, shared with mammalian Oct/POU transcription factors, to moderate responses to immune challenge, thereby increasing the tolerance to biotic stress.

Keywords: Antimicrobial peptides, Drosophila, Gene regulation, Host-pathogen interaction, Immune signaling, Innate immunity, NF-kappaB, Oct /POU transcription factors, Stress response

\footnotetext{
* Correspondence: ylva.engstrom@su.se

'Department of Molecular Biosciences, The Wenner-Gren Institute, Stockholm University, SE-106 91, Stockholm, Sweden

Full list of author information is available at the end of the article
} 


\section{Background}

All multicellular organisms rely on evolutionarily selected immune defense mechanisms for protection against faster growing unicellular organisms, such as bacteria, fungi and protozoa, as well as viruses. In addition, multicellular parasites are threats that have to be combated by protective and reactive defense systems. The innate immune system, which is present in all metazoans, possesses the necessary duality in preventing infections and conquering them effectively. The former involves constitutively operating defenses, which are always present and counteract the invasion and growth of microbes, while the latter is based on recognition of the invader(s) and the immediate activation of cascades of parallel immune reactions with the purpose of eradicating the invading organism.

Drosophila melanogaster is a powerful model organism to identify genes involved in the innate immune system and its regulation. Several signal transduction pathways are involved in transferring information from the extracellular site of infection to elicit changes in gene expression of effector molecules such as antimicrobial peptides (AMPs) and reactive oxygen species (ROS) (reviewed in [1-5]). There are several families of AMP genes in Drosophila and other insects, most of which have been shown to be highly up-regulated in response to infection, primarily a result of signaling via the Toll and Immune Deficiency (IMD) pathways. These pathways are activated through extracellular recognition of pathogenspecific signature molecules, such as bacterial peptidoglycans. Signal transduction promotes nuclear translocation of the key NF-kB/Rel transcription factors Dorsal-related immunity factor (Dif) and Relish, which bind to a large number of target genes and activate their expression. As a result of transcription factor binding, dramatic up- and down-regulation of immune-regulated genes occurs, as was shown in several whole genome expression analyses (reviewed in [2,3]). Immune response genes contain infection-induced response elements (IRE), which typically consists of a few nested $\mathrm{kB}$-like sites to which the NF-kB/Rel factors bind, linked with target sequences for tissue-specific GATA transcription factors [2]. Constitutive expression of ROS and of subsets of AMP genes is evident in epithelial linings of the digestive, respiratory and reproductive organs [4-6]. This is controlled via independent regulatory modules, of which a few have been identified and characterized $[7,8]$.

Negative control of immune defense genes is of paramount importance to prevent aberrant activation, and to attenuate the immune response once the infection is eliminated. It has been shown that negative feedback regulation occurs at several levels of both the Toll and IMD pathways (reviewed in [3-5]). Blocking this negative feedback regulation leads to sustained and/or stronger immune reactions [3,9]. Direct transcriptional repression of immune defense genes has also been demonstrated. The homeodomain transcription factor Caudal (Cad) was shown to act as a gut-specific negative regulator of AMP gene expression [10], and Drosophila AP1 and STAT proteins were reported to act as negative regulators of AttacinA (AttA) expression [11]. However, continuous presence of commensal microbes in the gut does not promote constitutive activation of large batteries of genes in an NF-kB-dependent manner, therefore additional transcriptional regulators must exist that repress or modulate the expression of immune defense genes.

The POU family of transcription factors constitutes a large group arranged in six subclasses (I to VI) [12,13]. The name POU has its origin from the founding mammalian members Pit-1 and Oct-1/Oct-2, and the Caenorhabditis elegans Unc-86 protein [14]. Five different POU protein genes are present in the Drosophila melanogaster genome, belonging to four of the POU family subclasses, indicating that this transcription factor family is evolutionarily ancient $[12,13]$. The Drosophila nub gene (also called POU domain protein 1 (Pdm1)) encodes a class II POU protein [15-17] and is a homolog of the human POU2F1/Oct-1 and POU2F2/Oct-2 genes [18], with which it shares considerable sequence similarity $[15,17]$. It has been predicted through genome annotation that the $n u b$ gene contains two independent transcription units (nub-RB and $n u b$ $R D)$ that each encode one specific protein variant: Nub-PB (104 kDa) and Nub-PD (65 kDa), which share the C-terminus, including the DNA-binding POU and homeodomains, but differ in their N-termini (Additional file 1). The Drosophila nub gene was originally identified as a viable mutation, $n u b^{1}$, caused by the insertion of a retrotransposon in the promoter region $[19,20]$ just upstream of the first exon of the $n u b-R D$ transcription unit (Additional file 1). The nub gene has been extensively studied for its roles in embryonic development, in differentiation of the central nervous system [21-24], and for normal growth and patterning of wings and legs [20,25-28]. More recently, immunostaining of Nub protein has been used as an enterocyte cell marker in the adult midgut [29], but the role of $n u b$ in these cells has not been defined.

Mammalian POU factors are well known regulators of genes involved in both innate and adaptive immune processes. Initially, the mammalian class II factors Oct-1 and Oct-2 (Oct1/2) were identified as activators of immunoglobulin gene expression in B cells via octamer sequences [30]. Although the involvement of Oct-1/2 in immunoglobulin gene expression and B-cell development could not be confirmed in knock-out mice [31,32], a large number of immunomodulatory and inflammatory genes have been shown to be targets of Oct- 1 in vitro and in cell-based assays (reviewed by $[33,34]$ ). 
We previously isolated cDNAs for three POU domain transcription factors in a yeast screen for novel regulators of immune response genes [35]. One of these, corresponding to the $n u b-R D$ transcript, activated expression of a Cecropin A1-luciferase (CecA1-luc) reporter in Drosophila cells, indicating that the Nub-PD protein is able to bind and regulate transcription from the $C e c A 1$ promoter. Here we show that Nub-PD acts primarily as a negative regulator of immune defense genes. This negative control of effector gene expression seems to be crucial in supporting a normal gut microbiome, as mis-regulated gene expression, due to a mutation in the $n u b$ gene, significantly changed the commensal gut flora. The elevated AMP gene expression was NF-kB/Relish-dependent, and could be blocked by transgene expression of Nub-PD, demonstrating its capacity to directly down-regulate expression of immune defense genes. We suggest that Nub protein serves a crucial role in suppressing aberrant immune and stress gene activation in healthy flies, thereby promoting immune homeostasis and tolerance to the commensal microflora.

\section{Results}

Nub is a negative regulator of NF-KB/Relish-driven immune gene expression

We isolated a $n u b \mathrm{cDNA}$ in a yeast screen for regulators of the Drosophila CecA1 gene [35]. This cDNA represented the $n u b-R D$ transcript, expressed from the $n u b$ gene, encoding the Nub-PD protein form (Additional file 1). In Drosophila cell culture transfection assays, the $n u b-R D$ cDNA was found to activate expression of a CecA1-luc reporter construct [35], supporting a possible role as a regulator of immune response genes. This result prompted an in-depth investigation of the in vivo role of this POU transcription factor in regulation of immune gene expression in flies.

To examine if Nub-PD is necessary for CecA1 expression in vivo, we analyzed the expression of a CecA1-lacZ reporter gene in uninfected flies, in a wild-type (wt) and $n u b^{1}$ mutant background. In homozygous $n u b^{1}$ flies (Additional file 1), expression of the $n u b-R D$ transcript is strongly reduced [20] and no Nub-PD protein is detected on immunoblots (Additional file 2B), whereas the Nub-PB protein is still expressed in the $n u b^{1}$ flies, confirming that $n u b^{1}$ mutation is specifically affecting the expression of the Nub-PD protein. Surprisingly, we observed prominent $\beta$-galactosidase $(\beta$-gal) reporter staining in the fat body of uninfected $n u b^{1}$; CecA1-lacZ flies (Figure 1B) compared to the control flies (Figure 1A), indicating that Nub-PD acts as a negative regulator of CecA1 expression in fat body. In addition, we observed strong CecA1-lacZ expression in the posterior midgut of $n u b^{1}$; CecA1-lacZ flies (compare Figure $1 C$ and D;
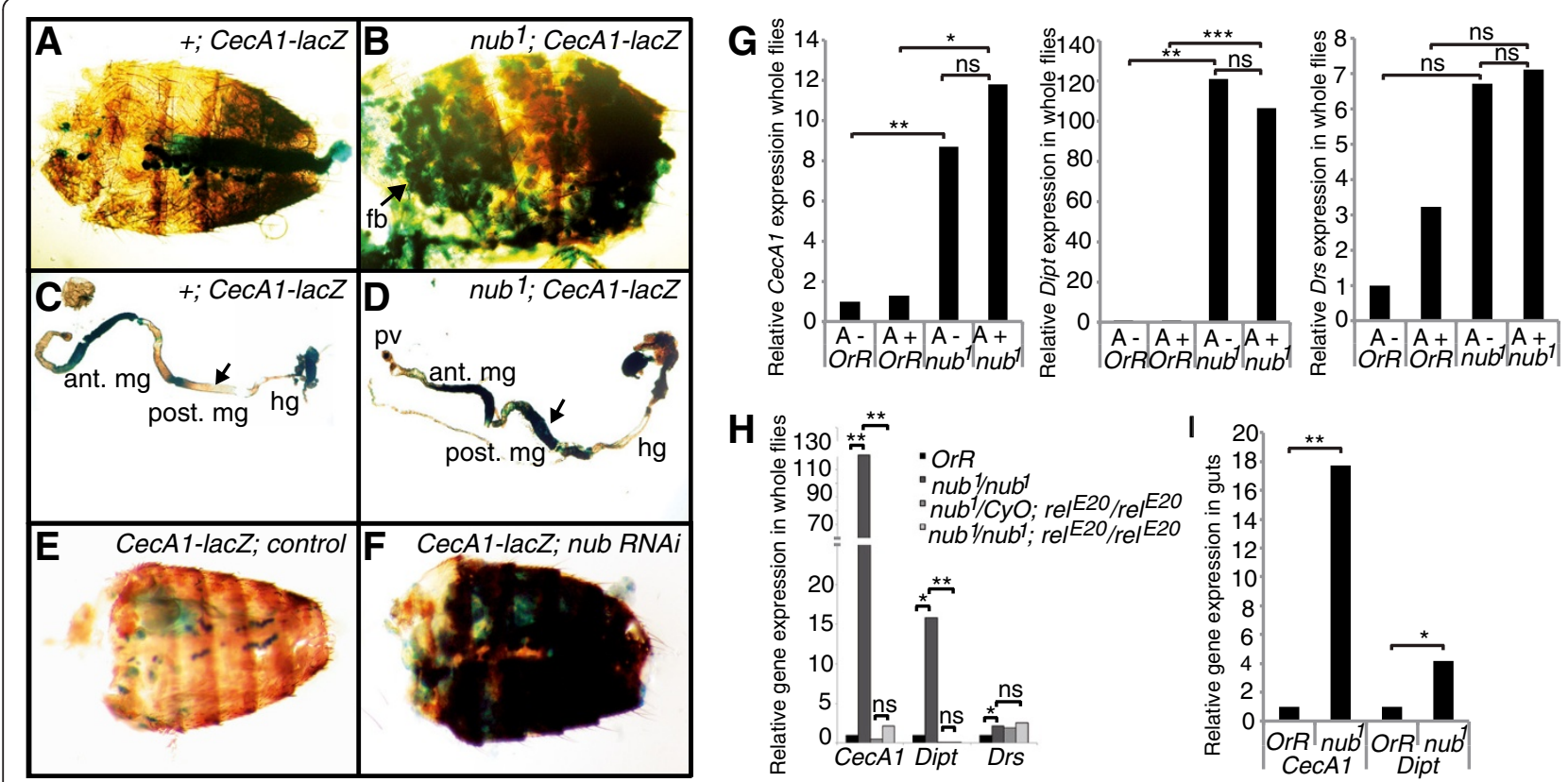

Figure 1 Antimicrobial peptide genes are over-expressed in uninfected $n \mathbf{u b}^{\mathbf{1}}$ flies. (A-F) Reporter $\beta$-galactosidase staining (CecA7-lacZ) in fat body and intestine of uninfected flies. Strong $\beta$-galactosidase expression was observed in fat body and posterior midgut (arrows) in nub mutant background (B, D) but not in wild type background $(\mathbf{A}, \mathbf{C}$ ). Elevated reporter gene expression was confirmed using RNA interference against nub in the fat body (CecA7lacZ/c564-Gal4;UAS-dsnub/+) (F), in comparison with control flies (CecA1-lacZ/c564-Gal4; +/+) (E). (G,H) Quantification of CecA1, Dipt and Drs mRNA levels in extracts of whole flies by RT-qPCR after pre-treatment with a cocktail of antibiotics (A+) or untreated control (A-) (G), and in Re $\left.\right|^{220}$ mutant background (H). (I) Quantification of CecA7 and Dipt mRNA levels by RT-qPCR in extracts of dissected intestines. The data are mean values; $n=3$ (G), $n=4$ (H,I). Statistical significance was calculated using paired $t$-test, ${ }^{*} P<0.05$, ${ }^{* *} P<0.01$, ${ }^{* * *} P<0.001$. fb, fat body; hg, hind gut; mg, midgut; pv, proventriculus; ns, not significant. 
arrows). To confirm that the elevated $\beta$-gal reporter staining is a consequence of the $n u b^{1}$ mutation, we used RNA interference (RNAi) to down-regulate $n u b$ transcript and protein. Expression of a hairpin construct, UAS-dsnub, driven by a fat body-specific Gal4 driver line (c564-Gal4) in transgenic flies, combined with the CecA1-lacZ reporter, promoted strong $\beta$-gal reporter staining in the fat body, compared to control flies (Figure 1E,F), strongly supporting that the $n u b$ gene is responsible for repression of $C e c A 1$ in healthy flies.

These results were further substantiated by analyzing the steady-state mRNA levels of a few AMP genes in extracts of whole flies and dissected guts by quantitative reverse transcriptase-PCR (RT-qPCR) (Figure 1G-I). Expression of CecA1 and Diptericin (Dipt) was significantly higher in $n u b^{1}$ than in wt flies (Figure 1G-1I), whereas Drosomycin (Drs) expression was only slightly increased (Figure 1G,H). To eliminate the possibility that the high level of AMP gene expression was due to the presence of an ongoing infection, and not the $n u b^{1}$ mutation, we treated flies with a potent cocktail of antibiotics prior to the analysis. Antibiotic-treated and untreated flies promoted similar levels of high CecA1 and Dipt expression (Figure 1G), indicating that Nub-PD was responsible for the repression of the expression of at least these two AMP genes, and also suggesting that activation, that is, derepression, occurs both in the absence and presence of commensal microbes in healthy flies. The CecA1 and Dipt genes are well-established targets of NF- $\mathrm{kB} /$ Relish transcriptional activation. Importantly, we found that the high steady-state expression of CecA1 and Dipt in uninfected $n u b^{1}$ flies is Relish-dependent, because it was significantly reduced in $\operatorname{Rel}^{E 2 O}$ mutants (Figure $1 \mathrm{H}$ ), whereas the small but significant up-regulation of Drs in $n u b^{1}$ flies was Relish-independent. Taken together, this indicates that Nub-PD acts as a transcriptional repressor of Relishdependent genes, possibly rendering these genes inactive in the absence of infection. In accordance with this assumption, we found that in the midgut, where Relish was previously shown to be constitutively cleaved and activated [10], CecA1-lacZ reporter staining was prominent in $n u b^{1}$ mutants (Figure 1D). In addition, CecA1 and Dipt mRNA levels were significantly elevated in dissected guts from $n u b^{1}$ flies compared to wt controls (Figure 1I). We conclude that Nub-PD acts as a negative regulator of Relish-dependent CecA1 and Dipt gene expression both in the gut and fat body. In response to bacterial infection, expression of CecA1, Dipt and Drs mRNAs was potently activated both in wt control and $n u b^{1}$ flies (Additional file 3). The expression levels of all three genes were reproducibly higher in $n u b^{1}$ compared to wt, suggesting a negative effect of the presence of Nub-PD in wt flies. However, due to large biological variation in transcriptional response to infection, especially in the $n u b^{1}$ mutant, the difference was not statistically significant (Additional file 3). In summary, our results indicate that Nub-PD is a negative regulator of the expression of at least two AMP genes, CecA1 and Dipt, in healthy flies. In response to infection, activation is dominant and repression is to a large extent alleviated, leading to strong immune gene expression in both wt and $n u b^{1}$ flies; at the same time, some de-repression was evident in $n u b^{1}$ mutants.

\section{The commensal bacterial load is significantly diminished} in guts of $n u b^{7}$ mutant flies

A possible consequence of the elevated AMP gene expression in guts of $n u b^{1}$ flies is that the numbers of commensal bacteria would be reduced. To test this we plated the gut content from individual flies on bacterial medium (Figure 2A) and found that whereas wt flies typically contained between $10^{3}$ to $10^{4}$ colony forming units per gut, no bacterial colonies grew from the guts of $n u b^{1}$ mutants $(n=15)$ (Figure 2), indicating that $n u b^{1}$ guts constitute a highly bacteriostatic environment. Although no colonies grew, we do not consider the guts of $n u b^{1}$ mutants to be completely devoid of bacteria, because amplification of bacterial 16S rRNA genes indicated that guts from $n u b^{1}$ mutants do contain bacteria (unpublished). Our conclusion is, however, that the numbers of live, cultivatable bacteria in guts of $n u b^{1} \mathrm{mu}-$ tants is strikingly diminished compared with that of wt flies. Thus, in the absence of a functional Nub-PD protein as in $n u b^{1}$ mutants, the gut microbiome is severely affected, most likely as a consequence of the increased expression of AMPs and possibly of other immune effector molecules.

\section{Nub-PD expression in $n u b^{1}$ flies is sufficient to restore a repressed status of target gene expression}

To investigate directly if Nub-PD acts as a transcriptional repressor of immune system genes, we overexpressed Nub-PD from a $U A S-n u b-R D$ construct in $n u b^{1}$ mutant background. We first analyzed expression of four AMP genes, CecA1, CecC, Dipt and AttC, in $n u b^{1}$; UAS-nub control flies, confirming strong overexpression of all four genes (Figure 3). A number of different Gal4 driver lines were then tested: the use of strong/ubiquitous drivers was lethal whereas tissuespecific drivers only promoted marginal $n u b-R D$ overexpression. Using a heat-shock promoter-driven Gal4 (UAS-nub-RD/hs-Gal4) it was also not possible to achieve high levels of $n u b$ mRNA expression after heatshock induction, although several different experimental regimes were tested. It is unclear why this is the case, but it may indicate that strong over-expression of $n u b$ $R D$ results in negative feedback regulation, most likely at the post-transcriptional level. However, approximately 


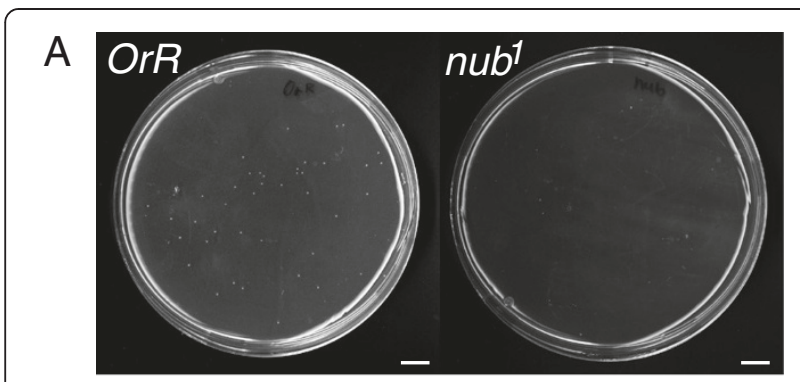

B

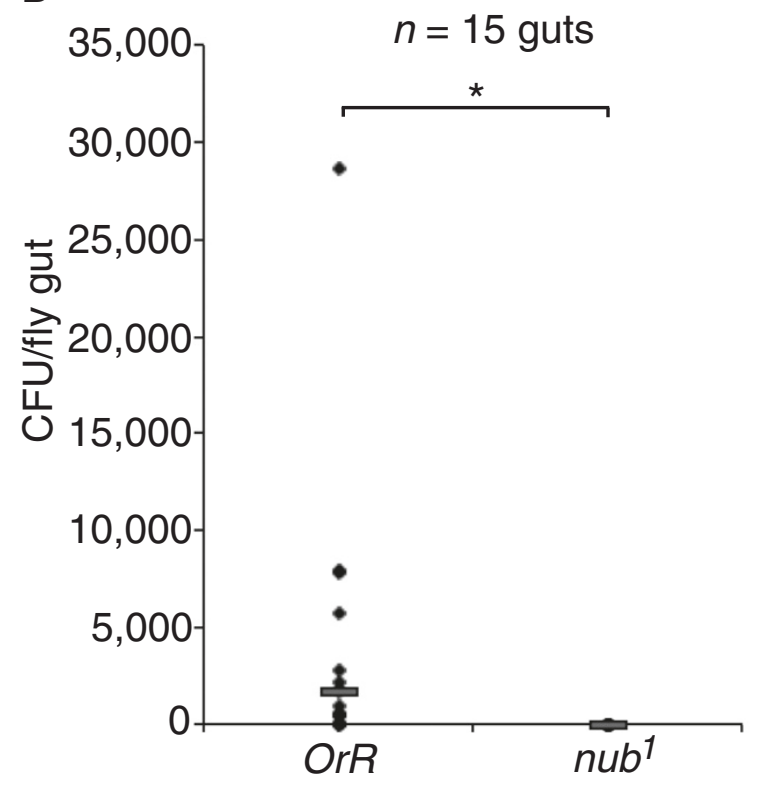

Figure 2 Homogenates of nub $b^{1}$ mutants contain a reduced number of platable bacteria. (A) Homogenates of dissected nub ${ }^{7}$ guts, spread out on standard lysogeny broth agar plates, contain a significantly reduced number of platable colony forming units compared to diluted homogenates from guts of OrR controls. (B) A total of 15 individuals guts per fly strain (n) were assayed and the median of the experiment is shown in the graph as a horizontal bar. Statistical significance was calculated using unpaired $t$-test, ${ }^{*} P<0.05$. Scale bar in $A=1 \mathrm{~cm}$. CFU, colony forming units.

10-fold over-expression of nub-RD was reached routinely using leaky expression from the same transgene combination (UAS-nub-RD/hs-Gal4) (Figure 3). This relatively moderate level of $n u b-R D$ over-expression in a $n u b^{1}$ mutant background (nub ${ }^{1}$; UAS-nub-RD/hs-Gal4) was sufficient to reduce CecA1, Dipt, CecC and AttC expression levels by $50 \%$ to $80 \%$ compared to $n u b^{1}$; UAS$n u b-R D / T M 3$ control flies (Figure 3), demonstrating that Nub-PD acts directly as a negative regulator of gene expression. It also confirms that aberrant expression of these immune genes in $n u b^{1}$ flies is truly due to the lack of a functional Nub-PD protein and not caused by the genetic background of the stock, as expression of UAS$n u b-R D$ was sufficient to restore the repressed status of

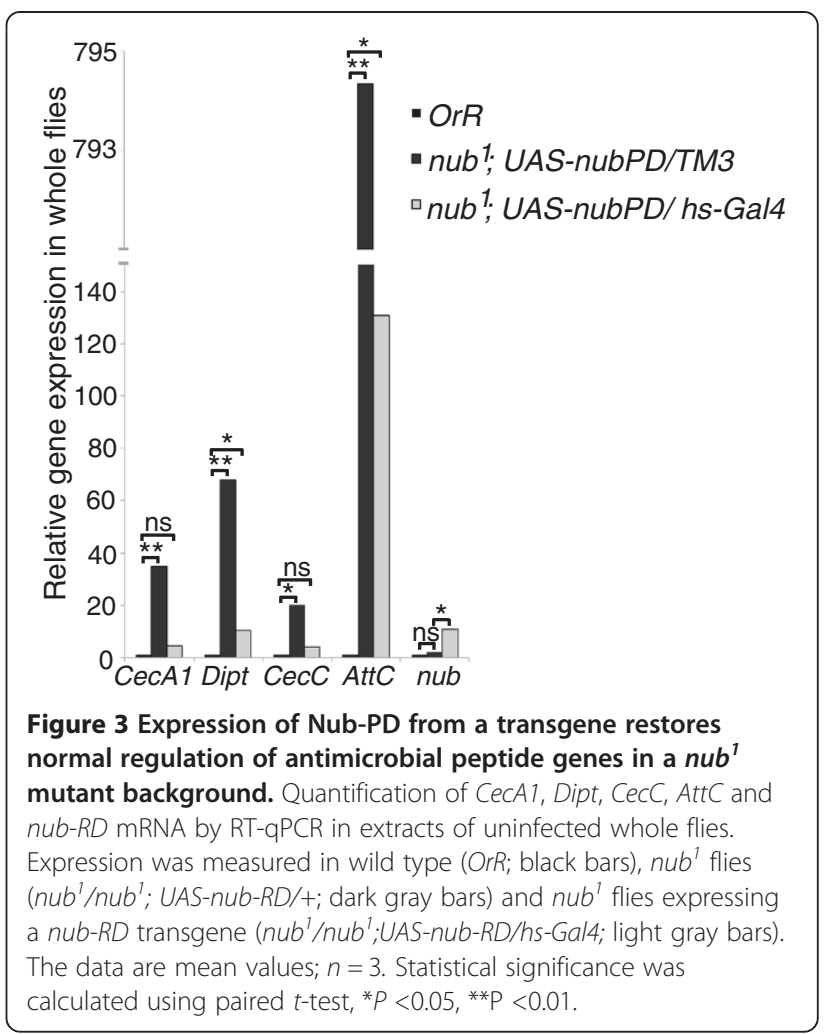

these genes. In infected flies, over-expression of $n u b-R D$ did not reveal a significant effect on the expression of CecA1 and Dipt (data not shown). As suggested above, this indicates that Nub-PD repression is relieved during infection.

\section{Repression of CecA1 by Nub-PD requires an upstream Oct} motif cluster

We have previously reported that the upstream regulatory region of the $\mathrm{Cec} A 1$ gene contains both positively and negatively acting regulatory elements, based on experimental evidence from promoter-reporter constructs in transgenic flies [36]. Similar to mammalian Oct1/2 transcription factors, Nub-PD has been shown to bind with high affinity to the Oct consensus motif $\left(\mathrm{ATGC}^{\mathrm{A}}\right.$ / $\left.{ }_{\mathrm{T}} \mathrm{AAT}\right)$ and to several closely related motifs [37-39] as well as more divergent non-Oct motifs [40]. We searched the CecA1 upstream region for these sequence motifs and found that it harbors an Oct cluster, containing three Oct consensus motifs, two Oct-like/nub motifs of the type present in the Drosophila choline acetyltransferase gene [38] and one variant of the latter overlapping with a $\mathrm{KB}$ site, located just $5^{\prime}$ of the IRE (Figure 4A). We did not find any sequences matching the Nub binding sites (non-Oct type) reported to be responsible for repression of the vestigial gene [40]. 

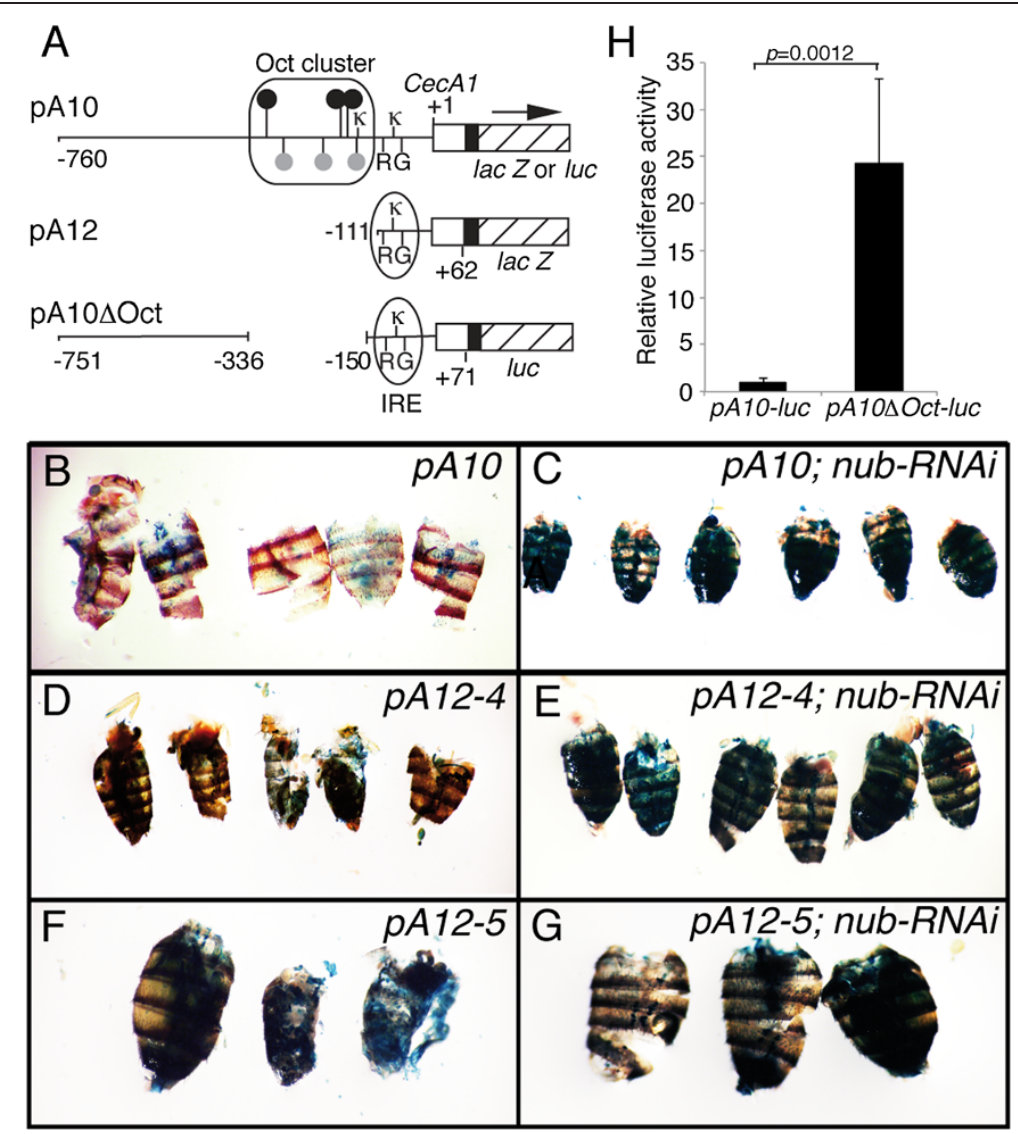

Figure 4 A distal promoter region of CecA1 is required for Nub-PD-dependent repression. (A) Schematic representation of the CecA1-lacZ and CecA1-luc constructs, either carried by transgenic Drosophila or used for cell transfections. The pA10 construct contains 760 or 751 bp of 5' upstream region from the CecA1 gene (horizontal line), and 62 or 71 bp of 5' UTR (open box) fused to a SV40 leader (filled box), providing a translational start site in frame with the Escherichia coli lacZ or luc coding sequence (hatched box) [7,41]. Numbers refer to positions relative to the transcription start site $(+1)$. Location of regulatory sequence motifs, as indicated by symbols and letters, is in scale. A previously characterized infection-induced response element (IRE) contains a kB-like site (k), GATA site (G) and R1 site (R), and one additional kB site is located just 5' of the IRE. A cluster of Oct sequence motifs (rectangle) contains several consensus Oct sequences (black circles) and Oct-like (gray circles) sequences (see Additional file 11 for sequences and exact locations). The pA12 construct contains 111 bp of 5' sequence including the IRE, but not the Oct cluster. The pA10 $\triangle \mathrm{Oct}$-luc construct has an internal deletion of the whole Oct cluster ( -336 to -150$)$ but is otherwise identical to the pA10-luc construct. (B-G) CecA1-driven $\beta$-gal staining in the fat body of abdomens from flies carrying either the pA10 construct (pA10 CecA7-lacZ / TM3) (B,-C) or the pA12 construct (pA12 CecA7-lacZ) (D-G). Two independent transgenic lines, pA12-4 (D,E) and pA12-5 (F,G) were used. (H) Transfection of mbn-2 cells with CecA1-luc constructs confirms that the Oct cluster region is involved in repression of the CecA1 promoter. The graph shows the mean values of relative luciferase activity and standard deviation $(n=6)$. Statistical significance was calculated using paired $t$-test, $P=0.0012$.

To investigate if the upstream region containing the Oct cluster is involved in repressing CecA1 expression in uninfected flies, we analyzed the expression of CecA1lac $Z$ constructs in transgenic flies carrying either a complete upstream region (pA10) or with a $5^{\prime}$ deletion, lacking the whole Oct cluster (pA12) (Figure 4A). Both constructs contain the IRE, which has previously been shown to contain necessary target sequences for NF-kB and GATA transcription factors [2], and to promote strong reporter gene expression in response to infection [36]. As a positive control, we analyzed the expression of CecA1-lacZ in flies in which nub had been downregulated by RNAi in the fat body (c564-Gal4; UAS- $d s n u b)$. This promoted strong reporter gene expression in the fat body of flies exposed to RNAi (Figure 4C) compared with the matched control flies, in which RNAi was not induced (Figure 4B). Two independent transgenic lines carrying the pA12 CecA1-lacZ transgene conferred strong reporter gene expression in abdominal fat body (Figure 4D,F), demonstrating that in the absence of the upstream region including the Oct cluster, the CecA1 promoter is constitutively active in the fat body. The staining was remarkably strong compared with the control pA10 CecA1-lacZ flies (Figure 4B), and similar to the pattern and reporter strength of pA10 CecA1-lacZ in $n u b$ RNAi flies (Figure 4C). It is important to note that 
the whole experiment was carried out with uninfected flies, confirming that the CecA1 promoter normally is repressed in healthy flies but aberrantly activated in nub-depleted flies. Expression of pA12 CecA1-LacZ was equally strong in abdominal fat body regardless of whether the flies had been exposed to nub-RNAi (Figure 4E,G) or not (Figure 4D,F). This indicates that Nub-PD primarily represses CecA1 expression via sequences present in the -760 to -111 bp region.

Next we created a luc reporter construct with an internal deletion of the Oct cluster region from -336 to -150 (pA10 0 Oct-luc) (Figure 4A), and analyzed its expression in transiently transfected Drosophila mbn-2 cells. Specific deletion of the Oct cluster promoted 25-fold higher expression levels compared to the $p A 10-l u c$ control (Figure $4 \mathrm{H}$ ), clearly demonstrating that the Oct cluster region acts as a negative cis-regulatory element.

In conclusion, deletion of the Oct cluster strongly enhances expression from the CecA1 promoter both in cell transfections and in vivo. In addition, deletion of the CecA1 upstream region that contains the Oct cluster leads to excessive CecA1-lacZ expression in a very similar manner as down-regulation of $n u b$ by RNAi, suggesting that Nub-PD regulates CecA1 by binding to the Oct cluster.

\section{Nub protein is expressed in fat body and midgut of uninfected flies}

It has previously been shown that $n u b$ is expressed in the developing central nervous system of embryos [23] and in wing, haltere and leg discs of third instar larvae [20]. Except for distinct localization in midgut enterocytes [29], it is not known if Nub protein is expressed in immunoresponsive tissues. We produced an affinity-purified peptide-specific antibody against Nub. Immunoblot experiments confirmed the specificity of the antibody and also validated the recent annotation of the $n u b$ gene to encode two proteins of 104 $\mathrm{kDa}(\mathrm{Pdm}-\mathrm{PB})$ and $65 \mathrm{kDa}$ (Nub-PD) (Additional files 1 and $2 \mathrm{~A}$ ). Strong immunostaining in wing and leg imaginal discs of third instar larvae confirmed localization of Nub protein in these tissues (Additional file 2C and unpublished). Immunostaining of adult tissues in cryosections of whole flies revealed that Nub protein is present in fat body (Figure 5A), midgut (Figure 5B) and testis (unpublished). This correlates well with reported expression of $n u b$ transcripts in adults [42]. Nub immunostaining was not restricted in its subcellular localization to either nucleus or cytoplasm, instead its nuclear-cytoplasmic localization varied in different regions of these tissues, possibly reflecting alternative states of transcriptional regulation. In conclusion, $n u b$ is expressed in immune-responsive tissues, such as fat body and midgut of healthy flies, which would enable Nub protein to act as a transcriptional repressor of immune genes in the absence of infection.

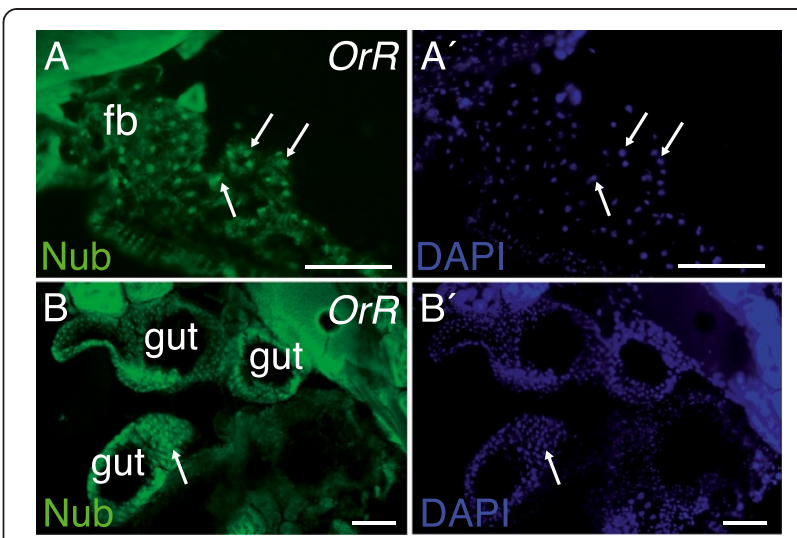

Figure $\mathbf{5}$ Localization of Nub protein in immunocompetent tissues of flies. (A,B) Immunostaining of Nub in frozen cryostat sections of uninfected OrR flies using a peptide-specific antibody directed against a common epitope of Nub-PB and Nub-PD protein. Nuclei stained with 4'-6-diamidino-2-phenylindole (DAPI) ( $\left.A^{\prime}-B^{\prime}\right)$ Cryostat sections (20 $\mu \mathrm{m}$ thick) of adults showing nuclear (arrows) and cytoplasmic and staining of Nub in fat body tissue $\left(A, A^{\prime}\right)$ and in cross-sections of midgut $\left(B, B^{\prime}\right)$. Scale bars, $100 \mu \mathrm{m}$.

Chromatin immunoprecipitation experiments show that Nub binds to antimicrobial peptide genes in vivo

In vitro DNA-protein interaction assays have previously demonstrated that Nub-PD binds with high affinity to the Oct sequence motif $[37,39]$. To analyze if Nub protein directly binds to the promoter regions of AMP genes, we carried out chromatin immunoprecipitation assays (ChIP). The PCR primers used for amplification were located to cover one or several Oct sites in the $5^{\prime}$ regions of analyzed genes (Figure 6A). Nub protein bound to the promoter regions of $C e c A 1, C e c C, A t t C$ and DiptA were isolated using the Nub-specific antibody (Figure 6B, lane 4). Importantly, Nub protein did not bind to the $A c t 5 C$ or to a non-transcribed intergenic region (Figure 6B, lane 4). Omitting the Nub antibody abolished the PCR product of all genes (Figure 6B, lane 2). As an additional control of specificity, we used an antibody against the C-terminal domain of RNA polymerase II, which is anticipated to neither bind to the relatively far 5 ' upstream regions analyzed here nor to AMP gene promoters in uninfected conditions [43]. As expected, the Cterminal domain of RNA polymerase II antibody did not bind to any of the AMP gene promoters but showed weak binding to the Act5C transcription unit (Figure 6B, lane 3). Phenol-chloroform extraction of isolated chromatin prior to immunoprecipitation abolished the PCR product in all samples (Figure 6C, lanes 7 and 8), validating that the immunoprecipitation requires the presence of protein bound to DNA. This ChIP demonstrates that Nub protein physically interacts with the promoter region of several AMP genes, strongly indicating that this physical interaction is responsible for repression of these genes in the absence of infection. 


\section{A}

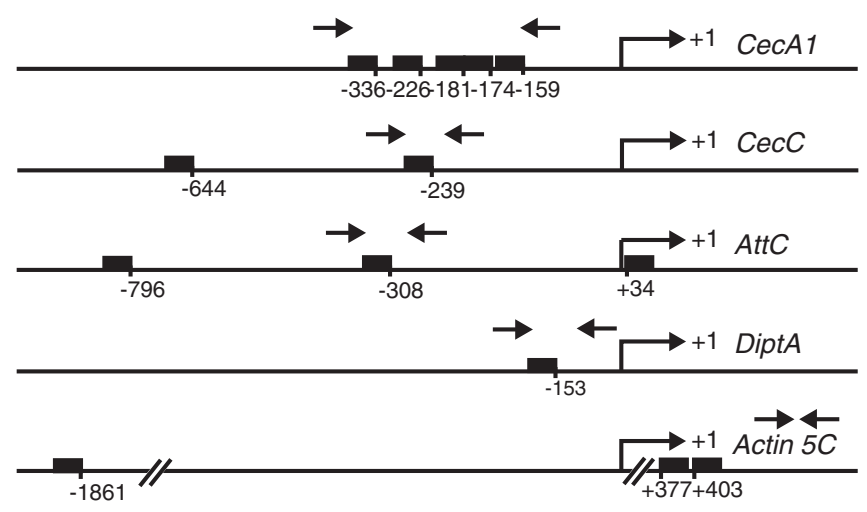

B
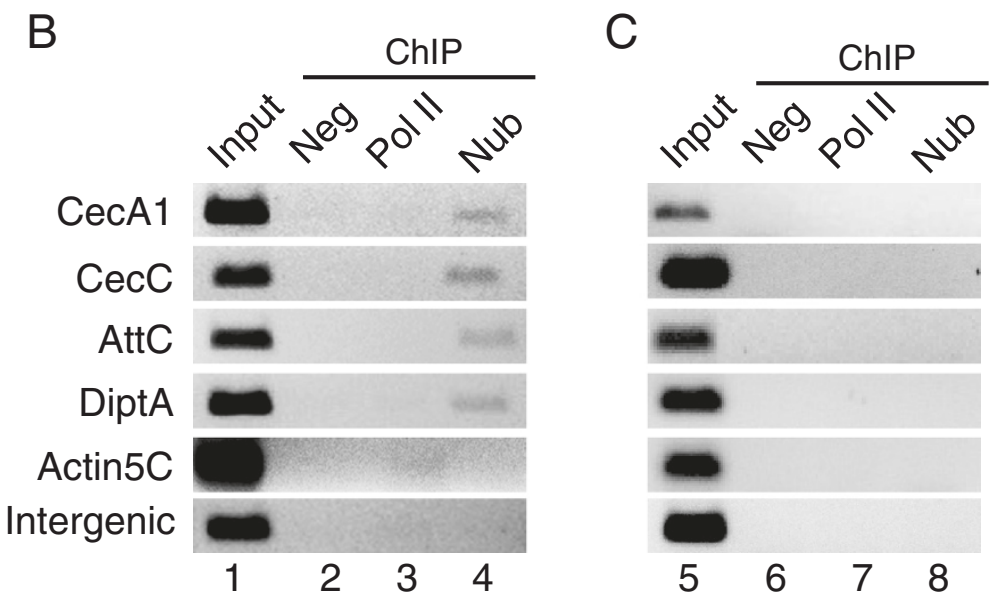

Figure 6 Nub binds to the upstream region of several antimicrobial peptide genes. (A) Promoter regions of CecA1, CecC, Dipt, AttC and Act5C, in which Oct and Oct-like-binding site(s) are represented by black boxes. The numbers below each box represents the position of the binding site in correlation to the transcriptional start site. The arrows indicate the forward and reverse primers. In the Act5C gene, two Octbinding sites are located in the first intron, while the primers bind to a region in the second intron. (B) Chromatin was extracted from whole OrR flies after fixation with formaldehyde and immunoprecipitation reactions were carried out with a peptide-specific Nub antibody (lane 4). Negative control immunoprecipitations were carried out in parallel, either without antibody (lane 2) or with an antibody against the C-terminal domain of RNA polymerase II (lane 3), which is not expected to bind to the upstream region of these genes in uninfected flies. Primers located in the transcribed region of $\mathrm{Act} 5 \mathrm{C}$ gene and in a non-transcribed region (intergenic region) were analyzed in parallel to assess the specificity of the chromatin immunoprecipitation, showing that RNA polymerase II, but not Nub, binds to the transcribed region of the Act5C gene, while neither protein binds to the non-transcribed region. (C) Control immunoprecipitation with chromatin, which was phenol-chloroform-extracted prior to fixation, showing the specificity of the protein-antibody interactions. As expected, no PCR products were observed with any of the antibodies.

\section{A large number of genes involved in immune system processes are up-regulated in $n u b^{1}$ flies}

To investigate if Nub-PD acts as a general repressor of immune gene transcription in healthy flies, we analyzed the global mRNA expression profile in $n u b^{1}$ mutants compared to wt flies by microarray analysis using Affymetrix Gene Chip Drosophila Genome 2.0 oligonucleotide arrays. Prior to mRNA extraction, the flies were dissected so that the two major immunoresponsive tissues, fat body (in carcass) and the digestive system (gut) were analyzed separately. The raw data were normalized, pre-processed and filtered to remove genes that were not expressed at a detectable level (see Methods).
A factorial map of principal component analysis reveales that the individual biological samples cluster together within their own sample group, showing that the replicates are well represented as a population (Figure 7A). The variance seen within each sample group is biologically expected as each sample is a pooled set of flies. Sample groups are firstly separated on the basis of tissue (gut or carcass) and secondly on strain ( $n u b^{1}$ or wt). No outlier samples were detected, hence all samples were included in further analysis. By comparing $n u b^{1}$ to the wt counterpart, either carcass or gut, we found 642 (carcass) and 961 (gut) transcripts to be differentially expressed by a fold change of two or higher. Out of 


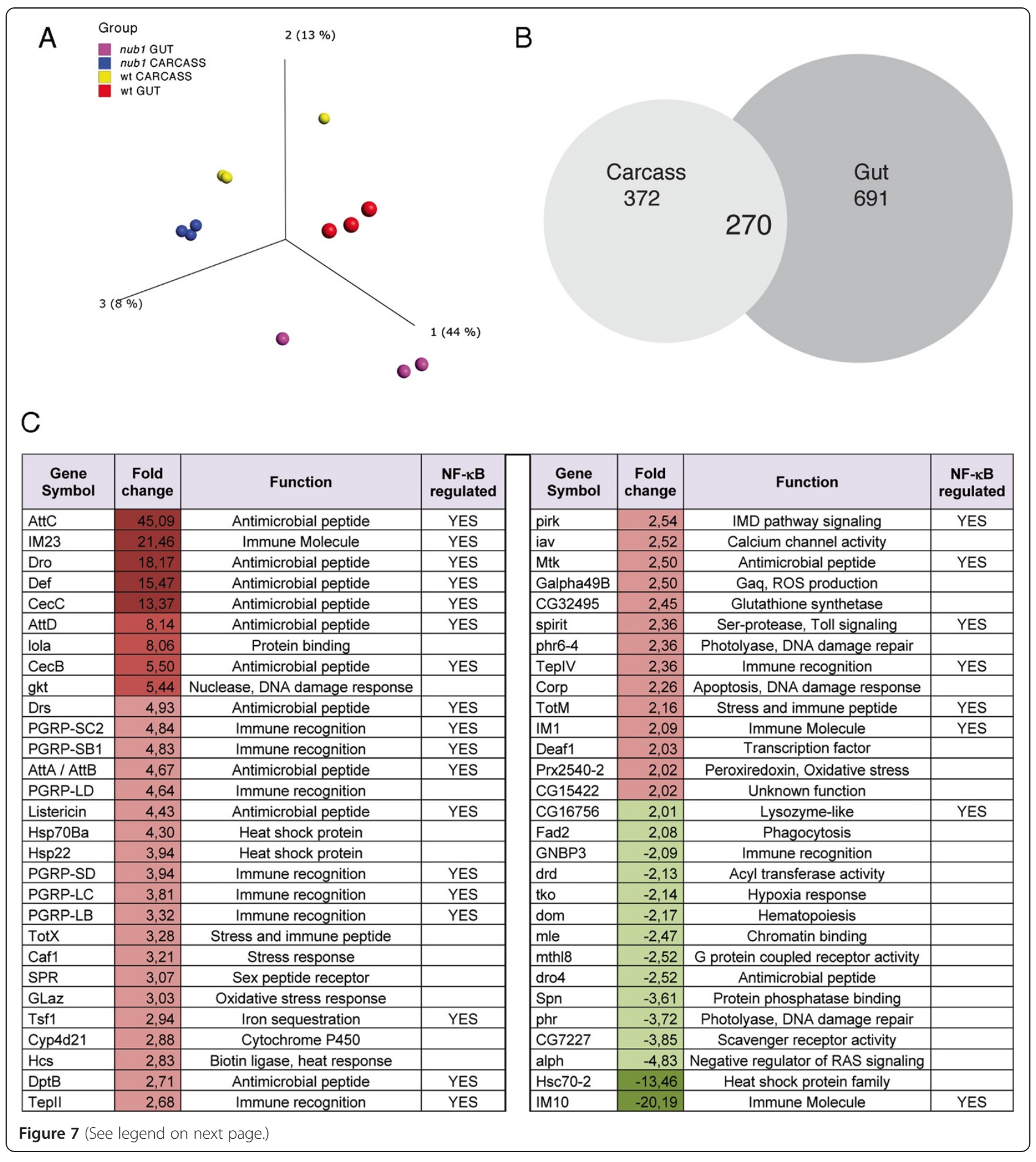


(See figure on previous page.)

Figure 7 Analysis of global mRNA expression profile in $n u b^{1}$ mutants compared to wild type flies. (A) A factorial map of principal component analysis was conducted using all transcripts found to be expressed over background signal in at least one sample group. Samples are colored group-wise. The percentage found for each component in the plot can be interpreted as the variation seen within and between the different sample groups. For the principal component analysis plots, the largest explainable variance is found in component 1, where $44 \%$ of the data's variation can be explained. This $44 \%$ is the biological difference found between the different tissues, gut or carcass. Component 2 with its $13 \%$ divides the data based on the biological variance explained by strain, that is, nub or wild type (wt). Component 3, with $8 \%$, biologically nuances the data even further. (B) Venn diagram showing the overlap of carcass and gut differentially expressed genes in nub ${ }^{7}$ compared to wild type after removing background and filtering for fold change >2. (C) List of 'Immune and Stress Response' genes up- or down-regulated in nub ${ }^{\dagger}$ mutants. Names and functions of the 58 genes constituting the enriched Biological Process cluster 'Immune system and Response processes' identified by a gene set enrichment analysis (Additional file 4) in carcass (without head and gut) from nub' mutants. Fold change (mean values) of mRNA levels is indicated by numbers and color-coded (red, up-regulation; green, down-regulation). Genes that previously have been shown to be targets of the NF-KB/Relish/IMD pathway or a combination of NF-KB/Relish/IMD and NF-KB/Dif/Toll pathways [44-46] are indicated by 'YES'.

these, 270 were differentially expressed in both carcass and gut (Figure 7B).

To further analyze the differentially expressed transcripts found in each sample type in $n u b^{1}$ versus wt, a gene set enrichment analysis was conducted identifying overrepresented Gene Ontology (GO) categories. The differentially expressed transcripts in carcass $n u b^{1}$ compared to wt are dominated by genes preferentially expressed in 'Immune and Response processes' (enriched with $P<0.0002$ ) (Additional files 4 and 5). Within this cluster of 58 genes (Figure 7C and Additional file 4) we find many well-characterized immune effector genes, such as AMPs and those involved in ROS production; immune recognition proteins, such as peptidoglycan recognition proteins (PGRPs); thioester proteins; and scavenger receptors. We also find genes involved in responses to abiotic stress, such as heat-shock proteins, detoxifying proteins, DNA damage repair, oxidative stress and wound healing. When we included $\operatorname{CecA} 1$ and Dipt, which were not present on the microarray but analyzed separately (Figures 1 and 3), we identified in total 60 immune defense and stress response genes that were differentially regulated in $n u b^{1}$ flies compared with wt. Out of these, $45(75 \%)(P<0.0002)$ were up-regulated in carcass samples in $n u b^{1}$ flies, supporting that Nub-PD primarily acts as a repressor of immune- and stressrelated genes. A large fraction of the 'immune defense genes' have previously been shown to be up-regulated by microbial infections in an NF- $\mathrm{kB} /$ Relish dependent manner (Figure 7C). We suggest that Nub-PD plays an important role in repressing immune defense genes, because the presence of commensal microbes will also lead to a low level of constitutive activation of NF- $\mathrm{BB}$ /Relish in uninfected conditions. Nub-PD will thereby increase tolerance to the presence of commensal microbes in healthy individuals.

Similar analyses of dissected gut samples also revealed significant enrichment $(P<0.00001)$ of genes involved in different types of 'Response processes' among the $n u b^{1}$ mis-regulated genes (Additional file 6). Within this combined cluster, consisting of 113 unique genes, $47 \%$ were up-regulated in $n u b^{1}$ gut compared to wt (Additional files 6, 7 and 8). Similar to the carcass samples, we identified genes involved in immune recognition, signaling and effector processes, as well as in abiotic stress responses among the $n u b^{1}$ mis-regulated genes (Additional file 7). Combining the sets of over-represented genes in carcass and gut samples involved in immune defense and other response processes, we found an overlap of 140 unique genes that are differentially expressed in $n u b^{1}$ flies compared to wt. A hierarchical cluster analysis of these genes (Additional file 9) shows that immune defense genes cluster together and are over-represented among genes that are up-regulated in $n u b^{1}$ mutants in either carcass or gut, or both.

From this global mRNA analysis, we conclude that numerous genes involved in immune defense reactions are abnormally expressed in $n u b^{1}$ mutant flies. Importantly, essentially all of the differentially regulated immune response genes have previously been experimentally validated for their functional roles in the immune system. In addition to genes clearly involved in the immune defense, the response processes clusters include genes with pertinent roles in cellular stress response processes, such as oxidative stress, hypoxia, heat-shock, DNA damage/repair and apoptosis. This suggests that Nub-PD may not only be involved in protecting flies against aberrant activation of immune system genes but also moderate responses to other types of cellular stress. We believe that Nub-PD acts as a modulator of immune responses, by preventing inappropriate expression of potent effector molecules. The array results also indicate that Nub-PD may play a role in regulation of stress responses, either directly or indirectly, but this assumption needs to be further validated.

\section{Genes involved in metabolism, development and differentiation require Nub-PD for normal expression}

Both in carcass and gut samples, GO processes related to metabolism and catabolism were strongly enriched (in carcass, $P<0.0001$; in gut, $P<0.00005)$ among the differentially expressed transcripts in $n u b^{1}$ mutants compared 
to wt (Additional files 4 and 6). By combining the sets of over-represented genes in carcass and gut samples, we found an overlap of 111 unique genes involved in metabolic and catabolic processes, and differentially expressed by a factor of at least two in $n u b^{1}$ flies compared to its wt counterpart. Of these genes, $48 \%$ were down-regulated in gut in $n u b^{1}$ mutants (Additional file 6), indicating an important role for Nub-PD in maintaining normal function in the gut and in metabolic homeostasis.

The role of $n u b$ in developmental processes during development of larval and adult structures, such as nervous system, wing and leg, is well documented [20,21,23,25-28]. Our gene expression analysis of $n u b^{1}$ mutants revealed that in the adult gut, there is differential expression of similar clusters of genes that previously have been linked to nervous system development (101 genes), development and differentiation of wing and legs ( 89 genes), and subgroups of these GO terms (Additional file 8). Again, there is much overlap between these groups with 80 unique genes shared between the GO biological processes 'Development and Differentiation' and 'Nervous System'. A large proportion of these genes cluster together (Additional file 10) and are down-regulated in $n u b^{1}$ gut samples, indicating that these genes normally require Nub-PD for their expression in the gut. Of these genes, many are known components of signaling pathways involved in patterning and differentiation of the nervous system in the embryo, and in wing and leg development during metamorphosis. This indicates that the same regulatory systems, involving Nub-PD, also operate in the adult gut, most likely during gut regeneration and differentiation of the gut epithelium.

\section{Genes that are mis-regulated in $n u b^{1}$ mutants contain Oct sequence motifs}

Having demonstrated that Nub protein directly binds to the upstream region of several AMP genes, we decided to perform a survey for Oct sites in the upstream region of genes that were differentially expressed in $n u b^{1}$ compared to wt flies (Figure 7C and Additional file 7). We focused our attention on genes belonging to $\mathrm{GO}$ terms related to immune defense, but also included a number of the most differentially expressed non-immune genes, and a few house-keeping genes as controls. In total, 60 genes were searched for the presence of the consensus Oct site $\mathrm{AT}(\mathrm{C}, \mathrm{G})(\mathrm{C}, \mathrm{G}, \mathrm{T}) \mathrm{AAA}(\mathrm{A}, \mathrm{T})$ and the Oct-like/nub target motif ATTCAAAT, present in the gene for Drosophila choline acetyltransferase [38]. We found a clear correlation between genes that were mis-regulated in $n u b^{1}$ mutants and the presence of Oct sites in the promoter and upstream region (Table 1 and Additional file 11), whereas three typical immune system genes (Dro6, Drslike and Bsk (dJNK/MAPK) whose expressions were not changed in $n u b^{1}$ mutant flies did not contain any Oct sites (Table 1). Four house-keeping or reference genes that did not confer any mis-expression in $n u b^{1}$ mutants were also analyzed, revealing only one Oct site in the distal promoter region of one of these genes. As has been mentioned above, of the genes that showed differential expression in $n u b^{1}$ versus wt flies, and of the genes analyzed here, $71 \%$ were up-regulated and out of those, all but two were found to contain at least one consensus Oct site. It is important to note, however, that at least six immune response genes that were down-regulated in $n u b^{1}$ gut tissue also contained Oct sites (Table 1 and Additional file 11), suggesting that Nub-PD regulates constitutive expression of some immune system genes in the gut via Oct sites. In conclusion, in silico analysis of gene promoters of genes that are misregulated in $n u b^{1}$ flies strongly indicates that Nub-PD directly controls a large group of immune system genes, as well as other genes, via Oct sequence motifs.

\section{Discussion}

Immune defense processes have to be instant and powerful to fight emergent infections efficiently and rescue the host. Minute concentrations of immune elicitors can communicate the presence of foreign organisms, which will lead to coordinated changes in expression of downstream effector genes. However, unprovoked activation of immune responses can be harmful because production of very potent biological effector molecules may cause damage to host tissues. Also, switching of gene regulatory programs may interfere with normal growth, development and other essential processes. Both signaling and gene expression need to be well controlled both before and after the acute stage of an infection, to regulate the rapid and powerful activation followed by attenuation of immune effector gene expression. In Drosophila, several negative regulators of immune regulatory pathways have been identified, especially for the IMD pathway $[3,4,9]$. Very few direct repressors of immune gene transcription have been identified, although it is generally acknowledged that dedicated transcriptional repressors are equally important as upstream regulatory pathways that limit production and activity of transcriptional activators [47]. The homeodomain protein Cad was shown to inhibit Relishdependent expression of AMP genes in the posterior midgut, and down-regulation of Cad by RNAi promoted over-expression of AMP genes [10]. Similarly, expression of AttA was reported to be inhibited by a repressosome complex, consisting of dAP-1, STAT92E and the High Mobility Group (HMG) protein Dsp1 [11]. The present work show that the Drosophila POU transcription factor Nub-PD is an important transcriptional repressor of immune defense genes.

Our work suggests that Nub-PD can act both as an activator and a repressor, but the mechanism for this 
Table 1 Gene, function and number of Oct and Oct-like/nub sites in the $\mathbf{5}^{\prime}$ upstream region

A. Oct sites in immune and stress response genes with $\geq 2$ fold change in carcass or gut (a)

\begin{tabular}{|c|c|c|c|c|c|c|}
\hline Gene & Computed gene number & Function & Fold change carcass & Fold change gut & Oct sites & Oct-like /nub \\
\hline CecA1 & CG1365 & Antimicrobial peptide & $>10 b$ & $>10 b$ & 3 & 2 \\
\hline $\mathrm{CecB}$ & CG1878 & Antimicrobial peptide & 5.5 & -1.4 & 1 & \\
\hline $\mathrm{CecC}$ & CG1373 & Antimicrobial peptide & 13 & b.b. & 2 & \\
\hline AttC & CG4740 & Antimicrobial peptide & 45 & 14 & 2 & 1 \\
\hline AttD & CG7629 & Antimicrobial peptide & 8.1 & -1.9 & & 1 \\
\hline Def & CG1385 & Antimicrobial peptide & 15 & 1.7 & 2 & \\
\hline Dpt & CG12763 & Antimicrobial peptide & $>10 b$ & $4 b$ & 1 & \\
\hline Dpt B & CG10794 & Antimicrobial peptide & 2.7 & -1.4 & 3 & \\
\hline Drosocin & CG10816 & Antimicrobial peptide & 18 & 4.4 & 1 & 1 \\
\hline Drs & CG10810 & Antimicrobial peptide & 4.9 & 1.1 & 1 & \\
\hline Dro2 & CG32279 & Antimicrobial peptide & b.b & 4.1 & 3 & \\
\hline Dro3 & CG32283 & Antimicrobial peptide & b.b & 2.2 & 1 & \\
\hline Dro4 & CG32282 & Antimicrobial peptide & -2.5 & -2.2 & 1 & \\
\hline Dro5 & CG10812 & Antimicrobial peptide & -1.2 & -7.9 & 2 & 1 \\
\hline Listericin & CG9080 & Antimicrobial peptide & 4.4 & -1.7 & 2 & \\
\hline Mtk & CG8175 & Antimicrobial peptide & 2.5 & 1.5 & 3 & 1 \\
\hline Anp & CG1361 & Antimicrobial peptide & b.b & 154 & 5 & \\
\hline LysX & CG9120 & Antimicrobial protein & b.b & -17 & 3 & \\
\hline IM23 & CG15066 & Immune molecule & 21 & 3.5 & 2 & 1 \\
\hline IM10 & CG18279 & Immune molecule & -20 & -26 & 2 & 1 \\
\hline PGRP-SC2 & CG14745 & Recognition & 4.8 & 1.1 & 3 & \\
\hline PGRP-SB1 & CG9681 & Recognition & 4.8 & 1.5 & 1 & \\
\hline \multirow[t]{2}{*}{ PGRP-LD } & CG32912-RB/RA/RD & Recognition & 4.6 & 1.8 & 1 & \\
\hline & & & & & 2 & \\
\hline PGRP-LC & CG4432 & Recognition & 3.8 & 2.9 & 2 & \\
\hline \multirow[t]{3}{*}{ PGRP-LB } & CC14704-RA/RC/RD & Recognition & 3.3 & 2.6 & 4 & \\
\hline & & & & & 5 & \\
\hline & & & & & 4 & 1 \\
\hline Tep ॥ & CG7052 & Recognition & 2.7 & 1.6 & 2 & \\
\hline Tep IV & CG10363 & Recognition & 2.4 & 1.8 & 4 & \\
\hline GNBP3 & CG5008 & Recognition & -2.1 & -3.9 & 3 & \\
\hline Sr-CIV & CG3212 & Scavenger receptor & b.b & 11 & 1 & 1 \\
\hline Galpha49B & CG17759-RD/RE & Signaling & 2.5 & 4.2 & 3 & 1 \\
\hline Pirk & CG15687 & Signaling & 2.5 & 4.3 & 5 & \\
\hline Prx2540-2 & CG11765 & Peroxiredoxin & 2.0 & -1.4 & 1 & \\
\hline Tsf1 & CG6186 & Iron sequestration & 2.9 & 2.1 & 2 & \\
\hline TotX & CG31193 & Stress peptide & 3.3 & 2.9 & 2 & \\
\hline TotM & CG14027 & Stress peptide & 2.1 & 2.2 & 3 & \\
\hline
\end{tabular}

B. Oct sites in immune genes and in house-keeping genes with no significant fold change in carcass or gut (a)

$\begin{array}{lllll}\text { Dro 6 } & \text { CG32268 } & \text { Antimicrobial peptide } & 1.2 & 1.0 \\ \text { Drs-like } & \text { CG32274 } & \text { Antimicrobial peptide } & 1.2 & 1.1 \\ \text { Bsk } & \text { CG5680 } & \text { JNK Signaling } & 1.1 & -1.3\end{array}$


Table 1 Gene, function and number of Oct and Oct-like/nub sites in the $\mathbf{5}^{\prime}$ upstream region (Continued)

\begin{tabular}{|c|c|c|c|c|c|c|}
\hline Gapdh1 & CG12055 & Glycolys & 1.2 & 1.1 & - & - \\
\hline Rpl32/rp49 & CG7939 & Ribosomal protein & -1.1 & -1.1 & 1 & - \\
\hline Act5C & CG4027 & Actin & 1.1 & 1.2 & - & - \\
\hline Aats-arg & CG9020 & Arginine tRNA syntase & 1.0 & -1.1 & - & - \\
\hline
\end{tabular}

Consensus sequence of Oct sites: AT(C,G)(C,G,T)AAA(A,T) and of the Oct-like/nub site: ATTCAAAT. Fold change indicates mRNA expression levels in gut and carcass, comparing wild type versus nub $b^{1}$ mutant, data taken from the microarray data in Additional files 5 and 8 unless other wise indicated. Non-reliable values due to concentrations below background have been removed. A longer list of analyzed genes including the positions of the Oct sites is presented as Additional file 11. ${ }^{\mathrm{a}}$ Location of sites within $2000 \mathrm{bp}$ from the transcription start site of respective gene or up to the nearest exone of an adjacent gene. ${ }^{\mathrm{b}}$ Data taken from RT-qPCR results (Figures 1 and 3). b.b., concentrations below background.

switch is not yet known. It has previously been shown, using transcription assays in Saccharomyces cerevisiae, that Drosophila Nub-PD binds to Oct sequence motifs to regulate transcription. Direct transactivation capacity was limited, suggesting that co-activators play a role [39]. We show that Nub-PD directly binds to proximal promoter regions of several AMP genes in chromatin prepared from whole flies. These promoter regions contain one or several Oct sites, of which some are overlapping with or located near IRE containing $\mathrm{kB}$ and/or GATA sites. It may be possible that the mechanism of Nub-PD repression involves direct competition for binding sequences. However, it may also depend on posttranslational modifications, interactions with co-factors and chromatin remodeling complexes.

We show that Nub protein is present in nuclei of fat body cells and in all regions of the gut in healthy flies (Figure 5), which makes it an ideal gate-keeper of immune gene expression. It was previously shown that the presence of peptidoglycan from commensal microbes leads to low levels of constitutive activation of NF- $\mathrm{kB} /$ Relish in the midgut [10]. While it can be debated whether the fat body is under continuous immune challenge or not, it has been shown that small peptidoglycan fragments shed from live or dead bacteria can cross barrier epithelia and activate a systemic immune response in the fat body [48-50]. Without protective mechanisms, this would lead to constant activation of immune and inflammatory reactions. Clearly, in the absence of Nub$\mathrm{PD}$, immune gene expression is over-active both in the gut and in the fat body; remarkably, this leads to serious consequences for the commensal gut flora, which are severely affected in $n u b^{1}$ mutants. We propose that Nub-PD plays an important role in several immunoresponsive tissues by suppressing immune activation and allowing the continuous presence of a commensal gut microbiome. The effects observed in $n u b^{1}$ flies are reminiscent of inflammatory diseases in mammals, in which gut homeostasis is disrupted and the immune system is constantly activated by the presence of the commensal gut flora. The present study indicates that negative regulation by $\mathrm{POU} /$ Oct transcription factor(s) are crucial in flies for suppression of immune activation, thereby promoting tolerance to the gut commensal flora. It has not yet been explored if negative regulation by $\mathrm{POU} / \mathrm{Oct}$ or other transcription factors normally promote tolerance to the commensal flora in healthy mammals, neither has the lack of such negative regulation been surveyed as a possible cause of chronic inflammatory disease establishment.

We show that at least 37 immune defense genes are over-expressed in flies lacking Nub-PD (Table 1 and Additional file 7), suggesting that it serves as a general repressor of immune gene expression in the absence of true infections. Most of the up-regulated genes encode direct effectors of the immune defense, such as AMPs and enzymes that synthesize ROS. This elevated expression of immune defense genes in the gut had a striking effect on the commensal gut flora of $n u b^{1}$ mutants. A majority of the up-regulated genes have previously been shown to be targets of the NF-kB/Relish/IMD pathway or a combination of NF- $\mathrm{kB} /$ Relish/IMD and NF- $\mathrm{kB} / \mathrm{Dif} /$ Toll pathways [44-46]. A few Toll pathway components were found to be down-regulated in $n u b^{1}$ mutants, such as Persephone, GNBP3 and WntD. It is important to note that genes encoding negative regulators of the immune response were either up-regulated in $n u b^{1} \mathrm{mu}-$ tants (PGRP-LB, PGRP-SC2, PGRP-SB1, Pirk) or not significantly changed (Dredd, Caspar, dUSP36, DNR1, $C a d, A P 1$, STAT92), demonstrating that, in the $n u b^{1}$ mutant, the increased expression level of a large number of effectors, such as AMP genes, is not an indirect effect due to decreased expression of the above mentioned negative regulators. We also did not observe any changed expression of co-activators that have been connected with immune pathway signaling or NF-kB-dependent expression (MED17 (dTRAP80), nejiere (dCBP), Akirin and Helicase89B), arguing against indirect effects via these factors. We confirmed that CecA1 and Dipt were also up-regulated in $n u b^{1}$ mutants after treatment with antibiotics (Figure 1G), excluding that excessive expression in $n u b^{1}$ mutants is caused by ongoing infections. The over-expression of these genes was, however, to a large extent NF- $\mathrm{B} /$ Relish-dependent, suggesting that NF- $\mathrm{kB} /$ Relish can be activated by the presence of peptidoglycan fragments from dying or dead bacteria. Our conclusion is that over-expression of immune genes in 
$n u b^{1}$ mutant flies is to a large extent IMD pathwayand NF- $\mathrm{kB} /$ Relish-dependent, but did not require presence of live bacteria. Taken together, these results strongly indicate that Nub-PD directly represses a large number of immune system genes in healthy flies.

The whole genome analysis in $n u b^{1}$ mutants revealed a comprehensive picture of the role of Nub-PD in regulation of genes involved in the fly's immune system. In addition, genes that belong to GO categories of stress response processes and metabolism or catabolism processes were also strongly enriched in $n u b^{1}$ mutants. This is highly reminiscent of results from gene expression profiling carried out with mammalian Oct-1 deficient cells, which showed a clear over-representation of genes involved in cellular, oxidative and metabolic stress responses. The Oct-1 deficient cells were hypersensitive to a number of different stress conditions, indicating that Oct-1 normally controls the activation of such effector genes [51]. In addition, Oct-1 responds to cAMP signaling in pancreatic and intestinal endocrine cells and may play a role in metabolic homeostasis [52]. Interestingly, Oct-1 has been shown to repress cytokine-induced, NF-kB-dependent expression of the genes for E-selectin and vascular cell adhesion molecules [53]. This was shown to be part of a system involving Oct-1 repression of NF- $\mathrm{kB}$ target genes involved in inflammatory processes, and to maintain vascular cells in a quiescent state. It is intriguing that both in insects and mammals, repression of NF-kB-dependent target genes by Nub-PD and Oct-1, respectively, seems to be a hallmark of balancing immune, inflammatory and stress responses. We suggest that these evolutionarily related transcription factors are ancient stress sensors that modulate responses and gene activity, and increase the tolerance to both biotic and abiotic stress.

\section{Conclusions}

This work sheds new light on the complex regulation of innate immunity. We show that the POU/Oct transcription factor Nub negatively regulates genes involved in immune responses. Nub-PD protein binds to upstream sequences and represses gene expression of several AMP genes in healthy flies. Importantly, flies that lack expression of Nub-PD protein have a significantly changed gut microbiome, indicating that the maintenance of a normal gut flora is dependent on negative gene regulation by Nub-PD. Whole genome expression data show that a large number of immune- and stress-regulated genes are normally controlled directly or indirectly by Nub-PD, while other groups of genes, involved in development and metabolic processes, require Nub-PD for their normal expression. This demonstrates that Nub, similar to mammalian POU/Oct proteins, is involved in both positive and negative gene regulation. Importantly, our experimental data provide support for the evolutionary conservation of innate immunity between flies and mammals. This conservation involves not only positive regulation by Toll receptor pathways and NF- $\mathrm{kB}$ transcription factors upon infection but also includes complex regulation by POU/Oct transcription factors to modulate gene activity in healthy subjects.

\section{Methods}

\section{Fly stocks, culture and infections}

The following fly strains were used: Oregon ${ }^{R}$ and $w^{1118}$ were used as wt controls and $n u b^{1} b^{1} \mathrm{pr}^{1}$ was used as the $n u b^{l}$ mutant in all experiments. Flies for overexpression of Nub-PD ( $w^{1118}$; UAS-nubRD) (described below and in Additional file 1) or down-regulation of Nub (UAS-dsnub) (VDRC \#6218) [54] were crossed with the Gal4 driver lines $c 564-$ Gal4 $\left(w^{1118} ; P\{\right.$ GawB $\left.\} c 564\right)$ (fat body) and $h s$-Gal4 ( $w ; P\{w[+m c]=$ Gal4-Hsp70.PB\}). Combinations with reporter strains CecA1-lacZ pA10 and pA12 [36] and with $\operatorname{Re}^{E 2 O}$ mutant flies [55] were obtained through conventional crosses. Flies were maintained in mixed female to male populations at $25^{\circ} \mathrm{C}$ with a $12 \mathrm{~h}$ light $12 \mathrm{~h}$ dark cycle. Recordings of AMP gene expression were done with exclusively females.

Germ-free flies were established by moving five-day old flies to sterile medium supplemented with a cocktail of antibiotics, and keeping them on this medium for at least seven days, as previously described [10]. The antibiotic cocktail was added during preparation of sterile culture medium to a final concentration of $100 \mu \mathrm{g} / \mathrm{ml}$ carbenicillin, $100 \mu \mathrm{g} / \mathrm{ml}$ neomycin, $50 \mu \mathrm{g} / \mathrm{ml}$ vancomycin, and $100 \mu \mathrm{g} /$ $\mathrm{ml}$ metronidazole. Control flies for this experiment were kept on sterile medium lacking antibiotics.

Microbial infections of flies were done with a mixture of over-night cultures of Gram-positive Micrococcus luteus and Gram-negative Enterobacter cloacae $\beta 12$, which were washed once and suspended in PBS (pH7). Five-to-ten day old flies were injected with $\leq 0.1 \mu \mathrm{l}$ bacterial suspension per fly using a glass capillary connected to a micro injector (TriTech Research, Los Angeles, CA, USA).

\section{Plasmids, generation of transgenic flies and cell transfections}

Construction of plasmids for the generation of transgenic flies carrying $U A S-n u b-R D$ was done using the Gateway ${ }^{\circ}$ Technology (Invitrogen, Carlsbad, CA, USA). Briefly, nub$R D$ cDNA was amplified from the expression plasmid pAct-Pdm1 [35] using Pfu DNA polymerase (Thermo Fisher Scientific, Waltham, MA, USA) and the following conditions: $95^{\circ} \mathrm{C}$ for 3 minutes; $95^{\circ} \mathrm{C}$ for 30 seconds, $60^{\circ} \mathrm{C}$ for 30 seconds, $72^{\circ} \mathrm{C}$ for 4 minutes, repeated 30 cycles; $72^{\circ} \mathrm{C}$ for 5 minutes. The purified PCR product was cloned into the $\mathrm{pENTR}^{\mathrm{m} \mathrm{m}} / \mathrm{D}-\mathrm{TOPO}$ vector using $\mathrm{pENTR}^{\mathrm{m} \mathrm{m}}$ Directional TOPO Cloning (Invitrogen) followed by recombination of the $n u b-R D$ cDNA into the pTW destination vector, 
(obtained from TD Murphy), using LR Recombination and the LR Clonase ${ }^{\mathrm{Tw}}$ enzyme mix (Invitrogen). P-element transformation of $w^{1118}$ flies was done according to standard protocols [56].

Deletion of the Oct cluster region in the CecA1 upstream region to create $p A 10^{\triangle} \mathrm{Oct}$-luc was done by inverse PCR with phosphorylated primers using the pA10-luc $[-751$ to +71$]$ construct [7] as starting material. Sequences of primers and details of the PCR protocol are described in the Additional file 12.

Cell transfections were done in Drosophila mbn-2 cells at $25^{\circ} \mathrm{C}$, using a calcium phosphate transfection kit (Invitrogen), as described previously [8]. The DualLuciferase ${ }^{\circ}$ Reporter Assay System (Promega, Fitchburg, WI, USA) was used to measure the different luciferase values, according to the manufacturer's instructions. Transfections were done with $1 \mu \mathrm{g}$ of $p A 10-l u c$ (gift from W-J Lee) or $p A 10^{\triangle}$ Oct-luc construct (this work) and $100 \mathrm{ng}$ of PolIII-Renilla luciferase (Addgene plasmid 37380) [57] (gift from N Perrimon) as internal reference, and mixed with carrier DNA to reach $10 \mu \mathrm{g}$.

\section{Cultivation of bacterial microflora}

Flies were anesthetized and sterilized in $70 \%$ ethanol for five minutes to eliminate bacteria on the fly surface. Individual guts were dissected under aseptic conditions and homogenized in $100 \mu \mathrm{l}$ sterile PBS using a plastic pestle. The whole volume of each $n u b^{1}$ gut homogenate was plated out on non-selective lysogeny broth agar plates $(1.5 \%[\mathrm{wt} / \mathrm{vol}]$ agar, $1 \%[\mathrm{wt} / \mathrm{vol}]$ tryptone, $0.5 \%$ [wt/vol] yeast extract, $1 \% \mathrm{NaCl}$ ), and each $\mathrm{OrR}$ gut homogenate was diluted $100 \times$ in sterile PBS prior to plating. The plates were incubated overnight at $37^{\circ} \mathrm{C}$. Because no colonies grew from $n u b^{1}$ homogenates, incubation at room temperature was also tested, which also did not produce any colonies.

\section{Antibody production, immunostaining and $\beta$ - galactosidase staining}

Antibodies against Nub-PD/PB were raised in rabbits against a synthesized peptide (C-QYKQEEDYDDANGG) (amino acids 119-132) conjugated to keyhole limpet hemocyanine carrier protein (Thermo Fisher Scientific, Waltham, MA, USA). The Nub peptide without the carrier protein was coupled to cyanogen bromide-activated sepharose $4 \mathrm{~B}$ according the manufacturer's protocol (Sigma-Aldrich, St Louis, MI, USA), and used for affinity-purification of the antisera.

Antibody staining of dissected larval and adult tissues was performed as described previously [58]. Cryostat sections of adults were prepared essentially as described in [59] with the following adjustments: cryostat sections (20 $\mu \mathrm{M}$ ) were cut using a cryomicrotome (Jung CM1800, Leica Microsystems GmbH, Wetzlar, DE).
Immunostaining of the sectioned tissues was done as described for dissected tissues. Primary antibody against Nub was used at $4 \mu \mathrm{g} / \mathrm{ml}$ and secondary antibodies were Alexa Fluor 488 conjugated goat anti-rabbit antibody $(1: 1,000)$ (Molecular Probes, Eugene, OR, USA). Specimens were analyzed in an Axioplan 2 fluorescence microscope (Carl Zeiss AG, Oberkochen, Germany), documented with a Hamamatsu Orca-ER digital camera (C4742-95) and processed with Axiovision Rel 4.8 software.

For analysis of CecA1-lacZ reporter gene expression, flies were dissected, fixed and stained for $\beta$-gal activity using 5 -bromo-4-chloro-3-indolyl- $\beta$-D-galactopyranoside as substrate, as described previously [36].

\section{Protein extraction and immunoblotting}

Protein extraction from the Drosophila cell line mbn-2 was done as previously described [60]. Extraction of 30 to 45 dissected intestines were done by homogenizing the tissues with a metal pestle dipped in liquid nitrogen and suspended in a non-denaturing buffer (20 mM 4-(2-hydroxyethyl)-1piperazineethanesulfonic acid, $\mathrm{pH} 7.9,0.56 \mathrm{M}$ potassium chloride, $0.2 \mathrm{mM}$ ethylenediaminetetraacetic acid, $1.5 \mathrm{mM}$ magnesium chloride, $2 \mathrm{mM}$ dithiothreitol, 25\% glycerol) supplemented with protease inhibitor according to the manufacturer (Roche Applied Science, Penzberg, Upper Bavaria, Germany). SDS-PAGE was done with 16 to $20 \mu \mathrm{g}$ of extracted protein per lane. After transfer to polyvinylidinefluoride membranes (Millipore Corporation, Billerica, MA, USA) the membrane was blocked in 10\% non-fat dried milk and incubated with anti-Nub antibody $(0.1 \mu \mathrm{g} / \mathrm{ml})$ overnight at $4^{\circ} \mathrm{C}$ and incubated with SuperSignal West Femto Maximum Sensitivity Substrate (Thermo Scientific) according to the manufacturer's instruction. Signal quantification was performed using computer software Image J [61].

\section{RNA extraction and quantitative RT-PCR analysis}

RNA extraction, DNase treatment, RT and PCR were carried as previously described [59]. For RT-qPCR analysis, total RNA was isolated from 10 to 30 flies (whole fly extracts) or from 45 dissected guts. When possible, primers and/or probes covered intron/exon boundaries to ensure specific amplification of cDNA; the sequences are given in Additional file 12. All samples were analyzed in triplicate (unless otherwise indicated), and the measured mRNA concentration was normalized relative to the control $R p L 32$ values. The normalized data were used to quantify the relative levels of a given mRNA according to comparative cycle threshold $\left(2^{-\Delta \Delta} \mathrm{CT}\right)$ analysis $[62,63]$. Statistical significance was calculated using paired $t$-test and $P$-values of $<0.05,<0.01$ and $<0.001$ were considered significant.

\section{Chromatin immunoprecipitation}

Chromatin was prepared from 20 female flies after manual homogenization and cross-linking with $4 \%$ formaldehyde 
as described previously [64] with the following adjustments: Chromatin fragmentation was done by sonication using a Bioruptur (CosmoBio-Diagenode, Liege, Belgium) for $4 \times 10$ minutes, to reach DNA size of 100 to $350 \mathrm{bp}$. Chromatin fragments were incubated with antibodies against Nub $(30 \mu \mathrm{g} / \mathrm{ml})$ or RNA polymerase II C-terminal domain $(10 \mu \mathrm{g} / \mathrm{ml})$ (Ab5408; Abcam, Cambridge, UK) and immune complexes were isolated using $50 \mu \mathrm{l}$ of a $50 \%$ mix of protein A- and protein G-dynabeads (Invitrogen). For immunoprecipitation experiments with naked DNA, phenol-chloroform extraction of proteins was carried out prior to cross-linking and sonication, using standard protocols. The isolated DNA fragments were amplified by PCR using primers specific for CecA1 (CG1365), CecC (CG1373), AttC (CG4740), DiptA (CG12763) and Act5C (CG4027) genes, and for the negative control primers were placed in a non-transcribed intergenic region in chromosome 3L. The PCR conditions were optimized to avoid saturation. Sequences of primers and details of the PCR protocol are described in Additional file 12.

\section{Microarray analysis, processing and extended analysis}

Total RNA was extracted as described [59] from dissected guts and from the rest of the fly minus gut and head (carcass) of 7- to 12-day-old female flies. Isolated RNA was further purified using RNAeasy (Invitrogen) according to manufacturer's instructions. Tissues from three independent pools of 20 flies were used and analyzed as biological replicates. Pre-processing of the rawdata (Affymetrix .cel files) was done according to the standard analysis pipeline at the Bioinformatics and Expression Analysis Core Facility at Karolinska Institutet, Huddinge, Sweden [65]. Briefly, .cel-files were imported into Affymetrix Expression Console, pre-processed and normalized using the MAS5 default pipeline. No outlier effects were revealed by quality control plots. The data discussed in this publication have been deposited in $\mathrm{Na}$ tional Centre for Biotechnology's Gene Expression Omnibus [66] and are accessible through GEO Series accession number [GSE44234] [67].

After pre-processing and normalization, two-group comparison t-tests were executed on respective sample groups ( $n u b^{1}$ and wt, as well as gut and carcass) to identify genes differentially regulated at either the 95\% confidence level $(P<0.05)$ or the $99 \%$ confidence level $(P<0.01)$. The raw data were normalized, pre-processed and filtered to remove genes that were not expressed at a detectable level (estimated background signal), leaving $44 \%$ and $41 \%$ of the transcripts expressed in wt carcass and gut, respectively. For the $n u b^{1}$ sample group, the distribution of expressed probe identities was $45 \%$ and $41 \%$ for the carcass and gut sample groups, respectively.

A factorial map of principal component analysis was executed on the whole expressed data by the program
Qlucore [68] on the differentially expressed (fold change >2). Gene set enrichment analysis to reveal enriched GO biological processes was performed using Cytoscape [69] and the plugin BiNGO [70]. The analysis was executed using the hyper-geometric test with Benjamini-Hochberg false discovery rate correction ( $P<0.05$ for gut; $P<0.01$ for gut and carcass).

Hierarchical clustering analyses were performed using Qlucore [68] on the GO hubs 'Immune System and Response Process' as well as extracted differentially expressed genes belonging to the GO clusters 'Development and Differentiation' and 'Nervous System'.

For the Venn diagram figures, a web-based program called Venny was used [71].

\section{Additional files}

\section{Additional file 1: The nub gene organization. \\ Additional file 2: Nub antibody specificity.}

Additional file 3: Expression of CecA1, Dipt and Drs mRNA is not significantly different in OrR and $n u b^{1}$ flies in response to bacterial infection.

Additional file 4: Gene set enrichment analysis of the 642 differentially expressed transcripts in nub ${ }^{7}$ carcass.

Additional file 5: Data set of gene set enrichment analysis for $n u b^{1}$ versus wild type comparison (carcass).

Additional file 6: Gene set enrichment analysis of the 961 differentially expressed transcripts in nub ${ }^{1}$ gut.

Additional file 7: List of 'Response process' genes up-or downregulated in $n u b^{7}$ mutants.

Additional file 8: Gene set enrichment analysis for $n u b^{7}$ versus wild type comparison (gut).

Additional file 9: Hierarchical clustering of 'Immune system and Response processes' genes.

Additional file 10: Hierarchical clustering of 'Development and Differentiation' and of 'Nervous System' genes.

Additional file 11: Presence of Oct and Oct-like sites in a selection of $n u b^{1}$ - differentially expressed genes.

Additional file 12: Primer/probes sequences and details of PCR protocols.

\section{Abbreviations}

AMP: Antimicrobial peptides; Att: Attacin; B-gal: B-galactosidase; bp: Base pairs; Cad: Caudal; Cec: Cecropin; CFU: Colony-forming Units;

ChIP: Chromatin immunoprecipitation; Dif: Dorsal-related immunity factor; Dipt: Diptericin; GO: Gene ontology; IMD: Immune Deficiency; IRE: Infectioninduced response elements; kDA: kiloDalton; NF: Nuclear factor;

PBS: Phosphate-buffered saline; PGRP: Peptidoglycan recognition proteins; RNAi: RNA interference; ROS: Reactive oxygen species;

RT-qPCR: quantitative reverse transcription polymerase chain reaction; UTR: Untranslated region; wt: wild type.

\section{Competing interests}

The authors declare that they have no competing interests.

\section{Authors' contributions}

WD, MMD, XT and YE conceived, designed and performed the experiments. $H U, A J$ and $A B$ designed and performed initial studies and experiments. JML carried out the extended microarray, statistical and bioinformatic downstream analysis. WD, MMD, JML and YE wrote the paper. All authors read, commented and approved the final manuscript. 


\section{Acknowledgements}

We would like to thank Bloomington Drosophila Stock Center and Vienna Drosophila RNAi Center for fly stocks, and Terence D Murphy, Won-Jae Lee and Norbert Perrimon for vectors and constructs. This research was supported by the Swedish Cancer Society (11-0577 to YE), The Swedish Research Council (B0494201 to YE) and a postdoctoral grant (80072501 to MMD).

\section{Author details}

'Department of Molecular Biosciences, The Wenner-Gren Institute, Stockholm University, SE-106 91, Stockholm, Sweden. ${ }^{2}$ Huddinge Genomics Core Facilities, Department of Biosciences and Nutrition, Karolinska Institute, SE-141 87, Huddinge, Sweden. ${ }^{3}$ Present address: Laboratories for Chemical Biology Umeå (LCBU), Umeå University, SE-901 87, Umeå, Sweden. ${ }^{4}$ Present address: Department of Pathology and Cell Biology, Columbia University, New York, USA.

Received: 14 June 2013 Accepted: 30 August 2013

Published: 6 September 2013

\section{References}

1. Lemaitre B, Hoffmann J: The host defense of Drosophila melanogaster. Annu Rev Immunol 2007, 25:697-743.

2. Uvell $H$, Engström $Y$ : A multilayered defense against infection: combinatorial control of insect immune genes. Trends Genet 2007, 23:342-349

3. Ganesan S, Aggarwal K, Paquette N, Silverman N: NF-kappaB/Rel proteins and the humoral immune responses of Drosophila melanogaster. Curr Top Microbiol Immunol 2011, 349:25-60.

4. Ryu JH, Ha EM, Lee WJ: Innate immunity and gut-microbe mutualism in Drosophila. Dev Comp Immunol 2010, 34:369-376.

5. Ferrandon D: The complementary facets of epithelial host defenses in the genetic model organism Drosophila melanogaster: from resistance to resilience. Curr Opin Immunol 2013, 25:59-70.

6. Davis MM, Engström Y: Immune response in the barrier epithelia: lessons from the fruit fly Drosophila melanogaster. J Innate Immun 2012, 4:273-283.

7. Ryu JH, Nam KB, Oh CT, Nam HJ, Kim SH, Yoon JH, Seong JK, Yoo MA, Jang $I H$, Brey PT, Lee WJ: The homeobox gene Caudal regulates constitutive local expression of antimicrobial peptide genes in Drosophila epithelia. Mol Cell Biol 2004, 24:172-185.

8. Junell A, Uvell H, Davis MM, Edlundh-Rose E, Antonsson A, Pick L, Engström $Y$ : The POU transcription factor Drifter/Ventral veinless regulates expression of Drosophila immune defense genes. Mol Cell Biol 2010 30:3672-3684

9. Zaidman-Remy A, Poidevin M, Herve M, Welchman DP, Paredes JC, Fahlander C, Steiner H, Mengin-Lecreulx D, Lemaitre B: Drosophila immunity: analysis of PGRP-SB1 expression, enzymatic activity and function. PLoS One 2011, 6:e17231.

10. Ryu JH, Kim SH, Lee HY, Bai JY, Nam YD, Bae JW, Lee DG, Shin SC, Ha EM, Lee WJ: Innate immune homeostasis by the homeobox gene caudal and commensal-gut mutualism in Drosophila. Science 2008, 319:777-782.

11. Kim LK, Choi UY, Cho HS, Lee JS, Lee WB, Kim J, Jeong K, Shim J, Kim-Ha J, Kim YJ: Down-regulation of NF-kappaB target genes by the AP-1 and STAT complex during the innate immune response in Drosophila. PLOS Biol 2007, 5:e238.

12. Bürglin TR, Ruvkun G: Regulation of ectodermal and excretory function by the C. elegans POU homeobox gene ceh-6. Development 2001, 128:779-790.

13. Larroux C, Luke GN, Koopman P, Rokhsar DS, Shimeld SM, Degnan BM: Genesis and expansion of metazoan transcription factor gene classes. Mol Biol Evol 2008, 25:980-996.

14. Herr W, Sturm RA, Clerc RG, Corcoran LM, Baltimore D, Sharp PA, Ingraham HA, Rosenfeld MG, Finney M, Ruvkun G, Horvitz HR: The POU domain: a large conserved region in the mammalian pit-1, oct-1, oct-2, and Caenorhabditis elegans unc-86 gene products. Genes Dev 1988 2:1513-1516.

15. Dick T, Yang XH, Yeo SL, Chia W: Two closely linked Drosophila POU domain genes are expressed in neuroblasts and sensory elements. Proc Natl Acad Sci U S A 1991, 88:7645-7649.

16. Billin AN, Cockerill KA, Poole SJ: Isolation of a family of Drosophila POU domain genes expressed in early development. Mech Dev 1991, 34:75-84.
17. Lloyd A, Sakonju S: Characterization of two Drosophila POU domain genes, related to oct-1 and oct-2, and the regulation of their expression patterns. Mech Dev 1991, 36:87-102.

18. Holland PW, Booth HA, Bruford EA: Classification and nomenclature of all human homeobox genes. BMC Biol 2007, 5:47.

19. Lindsley DL, Zimm GG: The Genome of Drosophila melanogaster. San Diego, CA: Academic Press, Inc:; 1992.

20. Ng M, Diaz-Benjumea FJ, Cohen SM: Nubbin encodes a POU-domain protein required for proximal-distal patterning in the Drosophila wing. Development 1995, 121:589-599.

21. Bhat KM, Poole SJ, Schedl P: The miti-mere and pdm1 genes collaborate during specification of the RP2/sib lineage in Drosophila neurogenesis. Mol Cell Biol 1995, 15:4052-4063.

22. Bhat KM, Schedl P: The Drosophila miti-mere gene, a member of the POU family, is required for the specification of the RP2/sibling lineage during neurogenesis. Development 1994, 120:1483-1501.

23. Yeo SL, Lloyd A, Kozak K, Dinh A, Dick T, Yang X, Sakonju S, Chia W: On the functional overlap between two Drosophila POU homeo domain genes and the cell fate specification of a CNS neural precursor. Genes Dev 1995, 9:1223-1236.

24. Bhat KM, Apsel N: Upregulation of Mitimere and Nubbin acts through cyclin E to confer self-renewing asymmetric division potential to neural precursor cells. Development 2004, 131:1123-1134.

25. Cifuentes FJ, Garcia-Bellido A: Proximo-distal specification in the wing disc of Drosophila by the nubbin gene. Proc Natl Acad Sci U S A 1997, 94:11405-11410.

26. Rauskolb C, Irvine KD: Notch-mediated segmentation and growth control of the Drosophila leg. Dev Biol 1999, 210:339-350.

27. Mirth C, Akam M: Joint development in the Drosophila leg: cell movements and cell populations. Dev Biol 2002, 246:391-406.

28. Natori K, Tajiri R, Furukawa S, Kojima T: Progressive tarsal patterning in the Drosophila by temporally dynamic regulation of transcription factor genes. Dev Biol 2012, 361:450-462.

29. Beebe K, Lee WC, Micchelli CA: JAK/STAT signaling coordinates stem cell proliferation and multilineage differentiation in the Drosophila intestinal stem cell lineage. Dev Biol 2010, 338:28-37.

30. Singh $\mathrm{H}$, Sen $\mathrm{R}$, Baltimore D, Sharp PA: A nuclear factor that binds to a conserved sequence motif in transcriptional control elements of immunoglobulin genes. Nature 1986, 319:154-158.

31. Corcoran LM, Karvelas M, Nossal GJ, Ye ZS, Jacks T, Baltimore D: Oct-2, although not required for early B-cell development, is critical for later Bcell maturation and for postnatal survival. Genes Dev 1993, 7:570-582.

32. Wang VE, Tantin D, Chen J, Sharp PA: B cell development and immunoglobulin transcription in Oct-1-deficient mice. Proc Natl Acad Sci U S A 2004, 101:2005-2010.

33. Phillips K, Luisi B: The virtuoso of versatility: POU proteins that flex to fit. J Mol Biol 2000, 302:1023-1039.

34. Kang J, Shakya A, Tantin D: Stem cells, stress, metabolism and cancer: a drama in two Octs. Trends Biochem Sci 2009, 34:491-499.

35. Junell A, Uvell H, Pick L, Engström Y: Isolation of regulators of Drosophila immune defense genes by a double interaction screen in yeast. Insect Biochem Mol Biol 2007, 37:202-212.

36. Roos $E, B j o ̈ r k l u n d ~ G$, Engström $Y$ : In vivo regulation of tissue-specific and LPS-inducible expression of the Drosophila Cecropin genes. Insect Mol Biol 1998, 7:51-62

37. Kalionis $\mathrm{B}$, O'Farrell $\mathrm{PH}$ : A universal target sequence is bound in vitro by diverse homeodomains. Mech Dev 1993, 43:57-70.

38. Kitamoto T, Salvaterra PM: A POU homeo domain protein related to dPOU-19/pdm-1 binds to the regulatory DNA necessary for vital expression of the Drosophila choline acetyltransferase gene. J Neurosci 1995, 15:3509-3518.

39. Prakash $\mathrm{K}$, Fang XD, Engelberg D, Behal A, Parker CS: dOct2, a Drosophila Oct transcription factor that functions in yeast. Proc Natl Acad Sci U S A 1992, 89:7080-7084.

40. Neumann CJ, Cohen SM: Boundary formation in Drosophila wing: Notch activity attenuated by the POU protein Nubbin. Science 1998, 281:409-413.

41. Engström Y, Kadalayil L, Sun SC, Samakovlis C, Hultmark D, Faye I: kappa Blike motifs regulate the induction of immune genes in Drosophila. J Mol Biol 1993, 232:327-333.

42. Chintapalli VR, Wang J, Dow JA: Using FlyAtlas to identify better Drosophila melanogaster models of human disease. Nat Genet 2007, 39:715-720. 
43. Gilchrist DA, Fromm G, dos Santos G, Pham LN, McDaniel IE, Burkholder A, Fargo DC, Adelman K: Regulating the regulators: the pervasive effects of Pol II pausing on stimulus-responsive gene networks. Genes Dev 2012, 26:933-944.

44. Irving P, Troxler L, Heuer TS, Belvin M, Kopczynski C, Reichhart JM, Hoffmann JA, Hetru C: A genome-wide analysis of immune responses in Drosophila. Proc Natl Acad Sci U S A 2001, 98:15119-15124.

45. De Gregorio E, Spellman PT, Tzou P, Rubin GM, Lemaitre B: The Toll and Imd pathways are the major regulators of the immune response in Drosophila. Embo J 2002, 21:2568-2579.

46. Buchon N, Broderick NA, Poidevin M, Pradervand S, Lemaitre B: Drosophila intestinal response to bacterial infection: activation of host defense and stem cell proliferation. Cell Host Microbe 2009, 5:200-211.

47. Payankaulam S, Li LM, Arnosti DN: Transcriptional repression: conserved and evolved features. Curr Biol 2010, 20:R764-R771.

48. Zaidman-Remy A, Herve M, Poidevin M, Pili-Floury S, Kim MS, Blanot D, Oh BH, Ueda R, Mengin-Lecreulx D, Lemaitre B: The Drosophila amidase PGRPLB modulates the immune response to bacterial infection. Immunity 2006, 24:463-473.

49. Gendrin $M$, Welchman DP, Poidevin M, Herve M, Lemaitre B: Long-range activation of systemic immunity through peptidoglycan diffusion in Drosophila. PLoS Pathog 2009, 5:e1000694.

50. Bosco-Drayon V, Poidevin M, Boneca IG, Narbonne-Reveau K, Royet J, Charroux B: Peptidoglycan sensing by the receptor PGRP-LE in the Drosophila gut induces immune responses to infectious bacteria and tolerance to microbiota. Cell Host Microbe 2012, 12:153-165.

51. Tantin D, Schild-Poulter C, Wang V, Hache RJ, Sharp PA: The octamer binding transcription factor Oct-1 is a stress sensor. Cancer Res 2005 65:10750-10758

52. Wang P, Wang Q, Sun J, Wu J, Li H, Zhang N, Huang Y, Su B, Li RK, Liu L, Zhang Y, Elsholtz HP, Hu J, Gaisano HY, Jin T: POU homeodomain protein Oct-1 functions as a sensor for cyclic AMP. J Biol Chem 2009, 284:26456-26465.

53. dela Paz NG, Simeonidis S, Leo C, Rose DW, Collins T: Regulation of NFkappaB-dependent gene expression by the POU domain transcription factor Oct-1. J Biol Chem 2007, 282:8424-8434

54. Dietzl G, Chen D, Schnorrer F, Su KC, Barinova Y, Fellner M, Gasser B, Kinsey K, Oppel S, Scheiblauer S, Couto A, Marra V, Keleman K, Dickson BJ: A genome-wide transgenic RNAi library for conditional gene inactivation in Drosophila. Nature 2007, 448:151-156.

55. Hedengren M, Asling B, Dushay MS, Ando I, Ekengren S, Wihlborg M, Hultmark D: Relish, a central factor in the control of humoral but not cellular immunity in Drosophila. Mol Cell 1999, 4:827-837.

56. Rubin GM, Spradling AC: Genetic transformation of Drosophila with transposable element vectors. Science 1982, 218:348-353.

57. Nybakken K, Vokes SA, Lin TY, McMahon AP, Perrimon N: A genome-wide RNA interference screen in Drosophila melanogaster cells for new components of the Hh signaling pathway. Nat Genet 2005, 37:1323-1332.

58. Patel NH: Imaging neuronal subsets and other cell types in whole-mount Drosophila embryos and larvae using antibody probes. Methods Cell Biol 1994, 44:445-487.

59. Davis MM, Alvarez FJ, Ryman K, Holm AA, Ljungdahl PO, Engström Y: Wildtype Drosophila melanogaster as a model host to analyze nitrogen source dependent virulence of Candida albicans. PLoS One 2011, 6:e27434.

60. Uvell $H$, Engström $Y$ : Functional characterization of a novel promoter element required for an innate immune response in Drosophila. Mol Cell Biol 2003, 23:8272-8281.

61. Schneider CA, Rasband WS, Eliceiri KW: NIH Image to ImageJ: 25 years of image analysis. Nat Methods 2012, 9:671-675.

62. Livak KJ, Schmittgen TD: Analysis of relative gene expression data using real-time quantitative PCR and the 2(-Delta Delta $C(T)$ ) Method. Methods 2001, 25:402-408.

63. Schmittgen TD, Livak KJ: Analyzing real-time PCR data by the comparative C(T) method. Nat Protoc 2008, 3:1101-1108.

64. Botelho SC, Tyagi A, Hessle V, Farrants AK, Visa N: The association of Brahma with the Balbiani ring 1 gene of Chironomus tentans studied by immunoelectron microscopy and chromatin immunoprecipitation. Insect Mol Biol 2008, 17:505-513.

65. The Bioinformatics and Expression Analysis Core Facility at The Karolinska Institute. [http://www.bea.ki.se]
66. Edgar R, Domrachev M, Lash AE: Gene Expression Omnibus: NCBI gene expression and hybridization array data repository. Nucleic Acids Res 2002, 30:207-210.

67. GEO Accession Display. [http://www.ncbi.n/m.nih.gov/geo/query/acc.cgi? acc $=$ GSE44234]

68. Qlucore software to explore and analyze microarray gene expression data sets. [http://www.qlucore.com/]

69. Saito R, Smoot ME, Ono K, Ruschainski J, Wang PL, Lotia S, Pico AR, Bader GD, Ideker T: A travel guide to Cytoscape plugins. Nat Methods 2012, 9:1069-1076.

70. Maere S, Heymans K, Kuiper M: BiNGO: a Cytoscape plugin to assess overrepresentation of gene ontology categories in biological networks. Bioinformatics 2005, 21:3448-3449.

71. Oliveros JC: VENNY. An interactive tool for comparing lists with Venn Diagrams. 2007 [http://bioinfogp.cnb.csic.es/tools/venny]

doi:10.1186/1741-7007-11-99

Cite this article as: Dantoft et al:: The Oct1 homolog Nubbin is a repressor of NF-KB-dependent immune gene expression that increases the tolerance to gut microbiota. BMC Biology 2013 11:99.

\section{Submit your next manuscript to BioMed Central and take full advantage of:}

- Convenient online submission

- Thorough peer review

- No space constraints or color figure charges

- Immediate publication on acceptance

- Inclusion in PubMed, CAS, Scopus and Google Scholar

- Research which is freely available for redistribution
C) Biomed Central 